\title{
Clustering Spam Emails Using Hadoop and FP-Trees
}

\author{
Danil Kirillov
}

January 5, 2017 


\section{Clustering Spam Emails Using Hadoop and FP-Trees}

By

Danil Kirillov

A thesis submitted to

the Faculty of Graduate and Postdoctoral Affairs

In partial fulfilment of the requirements for the degree of

Master of Computer Science

Ottawa-Carleton Institute of Computer Science

School of Computer Science

at

\section{Carleton University}

Ottawa, Ontario, Canada

January 2017

(C) Copyright by Danil Kirillov, 2017 
The undersigned hereby recommend to the Faculty of Graduate and Postdoctoral Affairs

Acceptance of the thesis

\title{
Clustering Spam Emails Using Hadoop and FP-Trees
}

\author{
Submitted by \\ Danil Kirillov
}

In partial fulfilment of the requirements for the degree of Master of Computer Science

Professor M. Barbeau (Supervisor)

Carleton University

January 2017 
To my mother and father. 


\begin{abstract}
Spam emails are a very current and ongoing issue. The sheer volume of spam emails makes the task of analysing them very tedious, for example, for campaign identification purposes. A spam email cluster is the abstraction of numerous spam emails that share common characteristics. Clustering spam emails aids by analysing them by bulk. One such clustering algorithm, proposed by Han et al. [1], revolves around using a data model called FP-Tree. We have implemented it in a practical system. We also made the algorithm harness the storage and processing capabilities of Hadoop, a distributed computing framework. The algorithm was also improved to produce less but more dissimilar clusters. Extensive experiments and evaluations were conducted. Results show that our implementation performs better in most circumstances than an original implementation by Dihn et al. [2]. This thesis presents the system design, improvements, and evaluation of our implementation of the FP-Tree based spam email clustering algorithm.
\end{abstract}




\section{Acknowledgements}

I would like to thank Dr. Michel Barbeau for the guidance he provided throughout the process of my thesis. Thank you for your patience, reviewing my thesis countless amount of times, and always providing valuable feedback. Thank you for always finding time for me.

I cannot forget to thank Frédéric Massicotte and Mathieu Couture who believed in me and encouraged me to take up and go through my graduate degree. Thank you for your advice, guidance, reviews, and feedback.

I would like to thank everyone at the Canadian Cyber Incident Response Centre for providing me with the resources and help needed for my thesis.

My mother and father, thank you for constant, never ending, support. Thank you for believing in me. 


\section{Contents}

1 Introduction 1

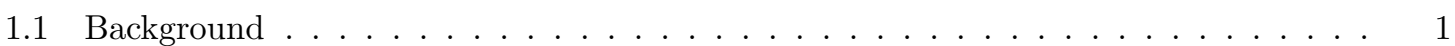

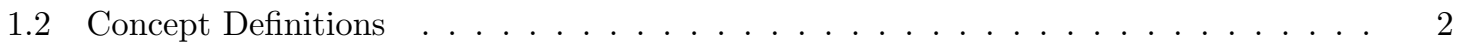

1.3 Problem Statement . . . . . . . . . . . . . . . . . . . . . . . . . . 3

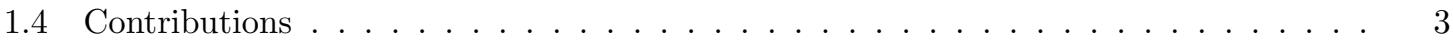

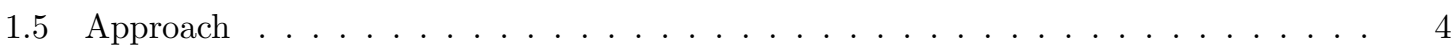

1.6 Performance and Clustering Results Summary . . . . . . . . . . . . . . . . . . 4

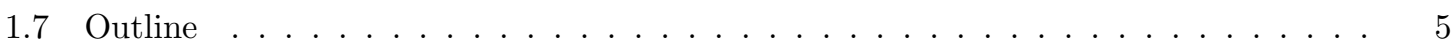

2 Literature Review $\quad 6$

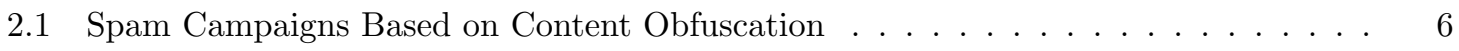

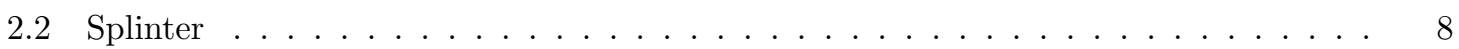

2.3 Spam Clustering Using Redirected URLs . . . . . . . . . . . . . . . . . . . . . 9

3 Hadoop and FP-Trees 12

3.1 Hadoop . . . . . . . . . . . . . . . . . . . . . . . . . . 12

$3.1 .1 \quad$ File System . . . . . . . . . . . . . . . . . . . . . . . . . . . 12

$3.1 .2 \quad$ Example Application . . . . . . . . . . . . . . . . . . . . . . . . . 14

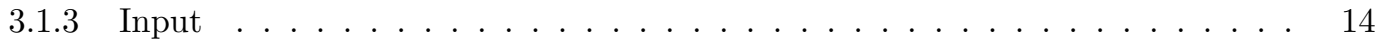


3.1 .4 MapReduce . . . . . . . . . . . . . . . . . . . . . . . 15

3.1 .5 Combiner and Partitioner . . . . . . . . . . . . . . . . . 17

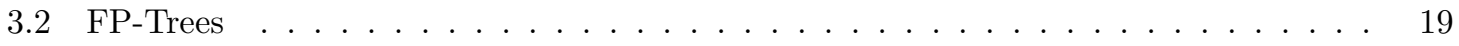

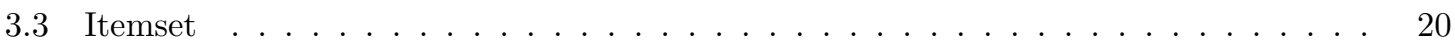

4 System Design 23

4.1 Spam Ingestion $\ldots \ldots \ldots \ldots \ldots \ldots \ldots \ldots \ldots$

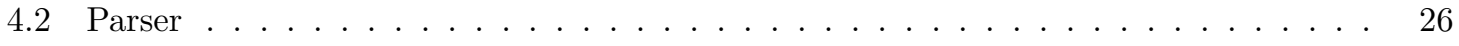

4.3 Tree Maker . . . . . . . . . . . . . . . . . . . . . . . . . . . . 28

$4.4 \quad$ Post Processing . . . . . . . . . . . . . . . . . . . . . . . . . . . 32

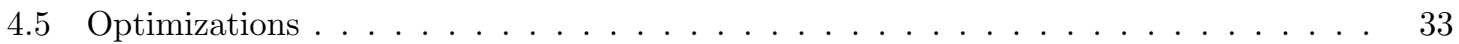

$\begin{array}{llr}5 & \text { Evaluation } & 36\end{array}$

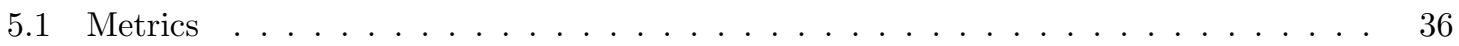

5.1.1 Jaccard and Overlap coefficients _ . . . . . . . . . . . . . . . . . . . . 37

5.1 .2 Silhouette coefficient . . . . . . . . . . . . . . . . . . 38

5.2 Methodology . . . . . . . . . . . . . . . . . . . . . . . 39

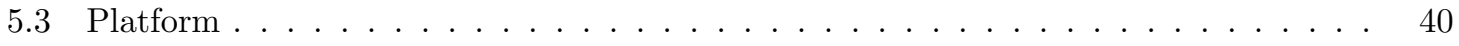

$\begin{array}{lll}6 & \text { Results } & 43\end{array}$

6.1 Datasets . . . . . . . . . . . . . . . . . . . . . 43

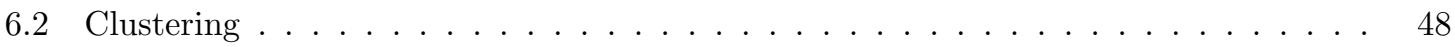

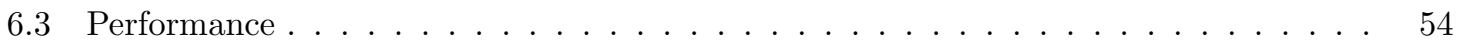

$\begin{array}{lll}7 & \text { Conclusion } & 57\end{array}$

$\begin{array}{ll}\text { A Collected Metrics } & 60\end{array}$

A.1 Clusters and Silhouette Scores for Larger Datasets . . . . . . . . . . . . . . . . . 60

A.2 Clusters and Silhouette Scores for Both Systems _ . . . . . . . . . . . . . . 61 
A.3 Performance Metrics . . . . . . . . . . . . . . . . . . . . . . 62

A.4 Parser MapReduce Pseudocode . . . . . . . . . . . . . . . . . . . . . 65

A.5 TreeMaker MapReduce Pseudocode . . . . . . . . . . . . . . . . . . . . 67

A.6 Splinter Pseudocode . . . . . . . . . . . . . . . . . . . . 70 


\section{List of Tables}

3.1 Example entities and itemsets . . . . . . . . . . . . . . . . . 19

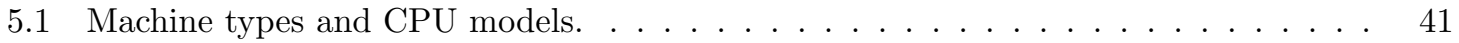

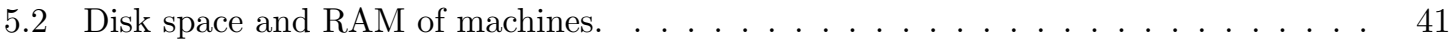

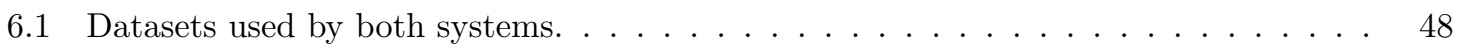

$6.2 \quad$ Larger datasets. . . . . . . . . . . . . . . . . . . . . . . . . . . . 52

A.1 Larger datasets metrics. . . . . . . . . . . . . . . . . . . . . 60

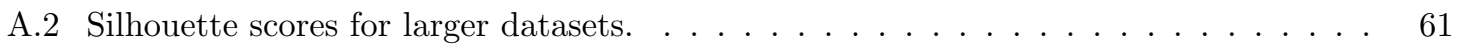

A.3 Cluster and graph metrics for Splinter and both variants of CAFE. . . . . . . . . 61

A.4 Silhouette scores for Splinter and both variants of CAFE. . . . . . . . . . . . 62

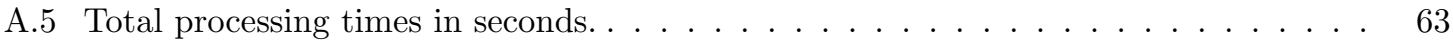

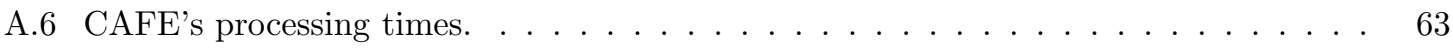

A.7 Splinter's processing times in seconds. . . . . . . . . . . . . . . . . . 64

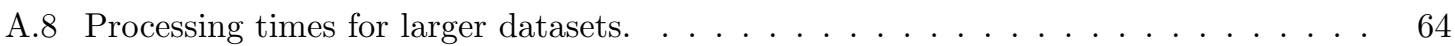




\section{List of Figures}

$3.1 \quad$ Example FP-Trees. . . . . . . . . . . . . . . . . . . . . . . . . . . . . . 19

3.2 Two similar example spam emails. . . . . . . . . . . . . . . . . . . . . . . 21

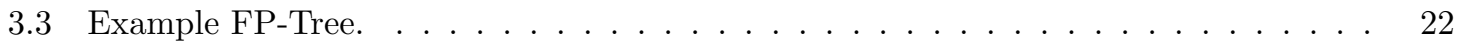

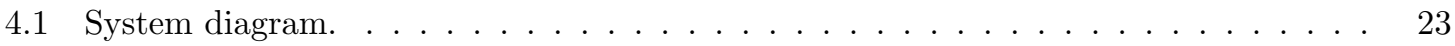

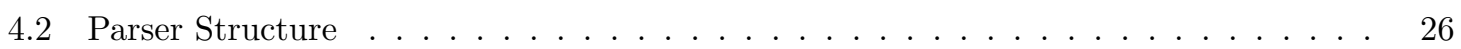

4.3 Tree Maker overview. . . . . . . . . . . . . . . . . . . . . . . . . . . . . . . . 29

6.1 Cutwail and Darkmailer FP-Trees. . . . . . . . . . . . . . . . . . . . 44

6.2 Gamut and Kelihos FP-Trees. . . . . . . . . . . . . . . . . . . . . 45

6.3 Lethic and Sendsafe FP-Trees. . . . . . . . . . . . . . . . . . . . . . . . 46

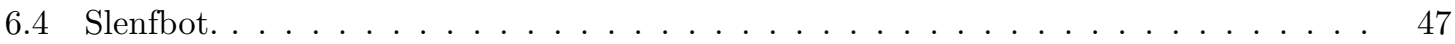

6.5 Number of clusters produced by each system for each dataset. . . . . . . . . . . . . . 49

6.6 Silhouette scores for clusters produced by each system for each dataset. . . . . . . . 50

6.7 Cutwail's Silhouette scores for merged and unmerged clusters. . . . . . . . . . . . 51

6.8 Number of clusters produced by each of the larger datasets by each variant of CAFE. 52

6.9 Silhouette scores for clusters produced by each of the larger datasets by each variant

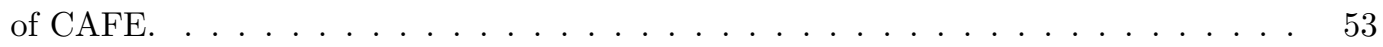

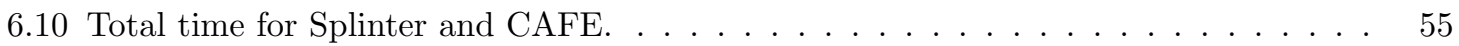




\section{LIST OF FIGURES}

6.11 Total time for parsing and clustering each of the larger dataset by each variant of CAFE 56 


\section{Chapter 1}

\section{Introduction}

Section 1.1 provides background information about spam campaigns. Section 1.2 presents relevant concept definitions. Section 1.3 describes the problem statement. Section 1.4 outlines the contributions made. Section 1.6 gives a brief summary of the results. Section 1.5 outlines the approach used to solve the problem. Section 1.7 provides an outline of the thesis.

\section{$1.1 \quad$ Background}

There has been a steady progress over the years in diminishing the large volumes of spam emails being sent out. Kaspersky Labs recorded that $68.3 \%$ of all email traffic accounted for spam in 2013 [3], $66.9 \%$ in 2014 [4], and $54.2 \%$ in 2015 [5]. While there is progress, there is still work that needs to be done to turn that $54.2 \%$ into $0 \%$.

Spam campaigns disseminate millions of emails across countless numbers of victims. They are still a very current and ongoing issue. Investigators across various security organizations are being tasked with looking into this issue, with the aim to reduce the number of people that fall victim to spam campaigns. Asking these investigators to analyze millions of spam emails each day to reveal information is a very tedious task. 
A spam campaign is defined as the dissemination of messages that share the same visible characteristics. The topic of this research is finding clusters in large volumes of emails. By identifying clusters, which are potential spam campaigns, investigators can accelerate into dealing with the source of them, thus reducing the number that fall victim to them.

\subsection{Concept Definitions}

While spam is a type of canned food, it does have a colloquial meaning in the digital world. Spam, or spamming, is repetition of things that are either irrelevant, inappropriate, or cause annoyance. This can come in various forms ranging from repeated advertisement, repeatedly sending or posting similar messages, to replay the same move in a video game. The term spam emails can then be described as repeatedly sent similar emails.

Similarity between spam emails can be seen in their subject lines, message bodies, embedded URL links, domains from URLs, or attachments. In fact, they could potentially be originating from the same spam campaign. Intuitively, one may know what a spam campaign is. It is a group of spam emails that have the same intent and share commonalities amongst themselves.

As easy as it is to intuitively define what a spam campaign is, there are subtle nuances in every definition. The definition that this thesis is using is closer to the one given by Dinh et al. [2]: a spam campaign is the dissemination of messages that share the same visible characteristics. This definition does not imply that we are aware of their intent, goals, or interrelations of actions. We are not participants or directors of spam campaigns, therefore we cannot truly know the intent or interrelations of actions as a campaign is going on. Judging whether it is truly a campaign or its intent is left to the investigators. The volume of data produced by a spam campaign can range from hundreds to hundreds of millions of emails. The system presented in this thesis proposes potential campaigns based on produced clusters. It makes no assumption on certainty or intent. The goal of this system is to aid investigators and not to replace them. 
Dissimilarity is the antonmy of similarity, which has been defined above. It is the differences between spam emails that are seen in their subject lines, message bodies, embedded URL links, domains from URLs, or attachments. The more dissimilar spam emails are to one another, the less likely that they originate from the spam campaign. Contextually, spam emails that are about selling luxery watches are different from those that are about jobs that pay thousands of dollars a day.

\subsection{Problem Statement}

Dinh et al. [2] have utilized Frequent Pattern-Trees (FP-Trees) for clustering spam emails and proposed a potential campaign detection algorithm, which they developed into a system called Splinter. We have conducted independent testing of Splinter, with approximately 1.2 million spam emails. We found that Splinter lacks scalability due to being a single machine and single thread system. How can the lack of scalability of Splinter be overcomed? We have observed that Splinter identifies a large number of clusters that are similar. How can constructed clusters be made more dissimilar?

\subsection{Contributions}

We revisited the algorithm presented by Dihn et al. [2]. We have implemented the revised algorithm utilizing the distributed computing framework Hadoop. Our system is named CAFE, short for cluster analysis of emails. Hadoop makes our system scalable while also increasing performance. The algorithm was further improved to produce less but more dissimilar clusters. To evaluate dissimiliarity between cluster, an evaluation methodology was devised. 


\subsection{Approach}

The system proposed in this thesis aims to identify clusters in large volumes of spam emails. It does this by processing the millions of spam emails that arrive each day, extracting useful information, and proposing spam email clusters. The cluster concept is used to identify potential spam campaigns. All of this is done in timely fashion, utilizing Hadoop.

The approach taken is to utilize Hadoop and the FP-Tree data model. Hadoop, with its distributed processing and storage capabilities, can handle large volumes of spam as well as ensuring that the system scales with the volume, it is described in Section 3.1. The FP-Tree data model is described in Section 3.2, which is used to identify clusters within the spam emails, that potentially represent spam campaigns. The algorithm has also been modified and tailored to run on the distributed computing framework Hadoop.

\subsection{Performance and Clustering Results Summary}

Following the approach described in Section 1.5, we have conducted expirements on CAFE. We have also conducted expirements with Splinter on the same datasets. The results are compared. An evaluation methodolody was devised to assess the clusters produced.

Utilizing the distributed computing framework Hadoop, our system was able to process larger datasets. The Hadoop cluster consisted of 30 machines, 26 of which were datanodes used for computing and storage. A full detailing of the platform used can be found in Section 5.3. Datasets processed by both CAFE and Splinter contained at most approximately 100 thousand spam emails. Datasets that could only be processed by CAFE contained starting from approximately 100 thousand to 3.7 million spam emails. Processing time has significantly improved, taking only hundreds of seconds by our system as opposed to thousands of seconds by Splinter. Processing time was also shown to scale with the amount of spam emails given.

We assessed the clusters produced for both intra-cluster and inter-cluster similarity using the 
Silhouette coefficient. Datasets used for clustering came from various botnets and across a three year time span. Results showed that our system produces less clusters that are more dissimilar. Chapter 5 explains the methodology in details. Chapter 6 describes the results in details.

\subsection{Outline}

The rest of the thesis is as follows. Chapter 2 is a literature review. Chapter 3 presents brief explanations about Hadoop and FP-Trees. Chapter 4 presents the system design and goes into details for each of its components. Chapter 5 presents the evaluation methodology that we use. Chapter 6 describes the dataset used for the evaluation, clustering, and performance of the system. Chapter 7 contains closing remarks. 


\section{Chapter 2}

\section{Literature Review}

This chapter goes over papers that attempt to overcome the problem of identifying spam email campaigns, or ways to cluster. Calais et al. [6] had done one of the earlier works with spam campaigns and FP-Trees. Their work is discussed in Section 2.1. Splinter, a system developed by Dihn et al. [2], is presented in Section 2.2. Instead of using FP-Trees, a different approach is taken by Shoeb et al. [7] that uses URLs, described in Section 2.3.

\subsection{Spam Campaigns Based on Content Obfuscation}

The work of Calais et al. [6] is one of the earlier works done on detecting spam email campaigns using FP-Trees. One of the problems tackled by Calais et al. [6] had been characterizing spam campaigns based on content obfuscation. Content obfuscation consists of various techiniques used by spammers to make obscure email content in order to avoid spam filters or to misslead users. One example of this is changing various letters in the subject from lower case to upper case. Another example is an HTML link showing a text link of one website address but having the href attribute set to another website address. They could also HTML encode parts of their message if the content type is HTML in order to avoid detection. The definition of a spam campaign given by them is a set of messages 
that have the same goal and employ the same obfuscation strategy, which comprises either content obfuscations or network exploitation. They employed FP-Trees to identify campaigns and content obfuscation strategies.

The item types that they used were the following:

1. Language - The human language of the content.

2. Message layout - Consisting of characters 'B' for a blank line, ' $U$ ' for a URL, and ' $T$ ' for a line of text.

3. Message type - The content type of the message, e.g., text/HTML, text/plain.

4. URL - The URLs were split into tokens by characters '/,'.', and '?'.

5. Subject - Email subject line.

They found that the FP-Tree was not tied to any current known pattern. Thus, as new types of spam campaigns emerged they were still able to cluster. Based on their items, they found that the language and message type were the two items that remained largely the same for large volumes of spam.

Based on URLs, they were able to determine three archetypes for spam campaigns. The first archetype is static campaigns. This type inserts the same constant URL into all spam emails in the campaign. The second archetype contains sub-campaigns within a campaign. These spam emails contain a set of different URLs that perhaps advertise different products. The last archetype is the random obfuscated campaign. These constantly change the URLs that are found in the spam emails.

A collection of spam emails was done by using honeypots. This allowed them to explore how spammers abused the network, among other lower-level details. Particularly the origin of spam emails along with the type of operating system and type of associated abuse. 


\subsection{Splinter}

Dihn et al. [2] created a software framework, named Splinter, that detects spam campaigns, analyses, and aids with investigation for large volumes of spam emails. It does so by consolidating spam emails into campaigns, labeling them, correlating between different data sources, and scoring campaigns based on criteria. Labeling and correlating directly aid the investigation by revealing characteristics of a campaign. Scoring campaigns allows investigators to focus on what is deemed to be the most havoc causing campaign.

Their approach is to use FP-Trees for detecting spam campaigns. There are key distinctions from Calais et al. [6]. First, the items that they chose to use are different. Second is the criteria for a campaign are different. Lastly, Dihn et al. [2] create the FP-Tree "on-the-fly", incrementally.

The items used by Splinter are the following: content type, character set, subject, email layout, URL hostname, URL path, URL parameters, and attachment names. To identify spam campaigns they use the following criteria:

1. A node that has a large number of children, with respect to a threshold.

2. The average frequency of children of that node is greater than a threshold.

3. In the path from that node to the root, there must be one node that does not have its item type in the none-obfuscated item list.

4. The number of leaves for the sub-tree at the node must be greater than a threshold.

Email layout in the case of a plain text message would consist of character ' $T$ ' representing a word, 'N' representing new line, and 'U' representing URLs. In the case of an HTML message, the HTML Document Object Modele of the message was used as the layout.

The none-obfuscated item list contains items that are not obfuscated by the spam senders. For instance, character set, is an item rarely obfuscated because it must be decoded successfully to have the email be read. 
They present a way to construct an incremental FP-Tree, which allows incoming spam emails to be added to the FP-Tree. However, the detection of spam campaigns would need to be re-run more frequently. Splinter also labels campaigns based on frequent words. Correlation is done based on WHOIS, passive DNS, geo-location, and additional malware data. They provide a way to score campaigns based on weighted criteria, such as hostnames resolving to a domestic country.

Text similarity was done between messages in each spam campaign. W-Shingling and Jaccard coefficient, Context Triggered Piecewise Hashing, and Locality-Sensitive Hashing were used to produce three scores per cluster. They all showed about half of the clusters scoring well and about half scoring poorly.

Dihn et al. [2] stated that it takes 40 minutes to parse the spam emails for a month, i.e., 100,339 spam emails. An additional one minute and a half was needed to produce campaigns. For a year of spam emails, containing 678,095 spam emails, it takes 3 hours to parse and 5 minutes to produce campaigns.

A copy of Splinter was given to us for testing purposes as well as internal documentation associated with it. Further claims in this thesis are based on the copy of Splinter received. Pseudocode for Splinter can be found in Appendix A.6. The copy provides a way to produce incremental FP-Trees. A Python script was needed to parse spam emails into an OrientDB, a distributed multi-model database. Another Python script was ran to detect spam clusters for a given time span. After that, additional scripts could be ran to score and label clusters. Algorithm 1 in Appendix A.6 shows the pseudocode for the script that parses. The pseudocode for the script that detects spam clusters is found in Appendix A.6 Algorithm 2 and the accompanying Procedure IdentifyCampaigns.

\subsection{Spam Clustering Using Redirected URLs}

Shoeb et al. [7] aim to reduce the effort needed to analyse the large volumes of spam emails by clustering them into campaigns. They use the redirected URLs generated from the embedded URLs 
found in spam emails as the primary source of clustering. This suggests that a significant amount of URLs that are embedded redirect to the same URL in the end. Subjects are used a secondary source of clustering.

The attributes that they use are the following:

1. Subject - Subject line of the email.

2. Sender IP - IP address of the machine that sent the email.

3. Machine - The web address of the machine that is used in the embedded URL.

4. Path - A path to a particular page on that machine.

5. URL - Concatenation of the machine and path.

The machine attribute can be seen as the hostname item from other papers. The path attribute also being one of the common items among the paper. The URL attribute described as the concatenation of machine and path attributes is in essence the original embedded URL that is found in the spam email. This URL is the one that will generate the redirected URLs.

An outline of algorithm presented in [7] is as follows:

1. Each spam email is considered to be a cluster of size 1 .

2. Merge all spam emails that redirect to the same URL into one cluster.

3. Merge each pair of clusters that contain a subject in common.

4. Merge each pair of clusters that contain a URL with a common domain.

5. Remove clusters with a size lower than the set threshold.

Step 1 is the starting point. The spam emails have already been parsed. Each is an individual cluster. On step 2, the redirect URLs are generated from the embedded URLs found in spam emails from step 1. The result of step 2 is a set of clusters based on the redirect URLs, as well as clusters 
consisting of one email that have not been merged by redirected URLs. Step 3 merges clusters based on subject, checking if each pair of clusters have at least one common subject in order to merge. At the end of step 3, the clusters created during step 2 have been merged even further. New clusters are created based solely on subjects. Step 4 uses domains of URLs within clusters as the attribute that is merged on. Each pair of clusters is checked for containing at least one common domain in order to merge. This is the last step. It merges and creates the final clusters. Step 5 deletes clusters that do not contain enough emails based on a threshold.

This approach is vastly different from using FP-Trees. It is possible to mimic this approach by creating an FP-Tree using three item types: redirected URLs, subjects, and domains. An FP-Tree created using those three item types should have the redirected URL be the closest to the root since it is suggested that a significnt amount of URLs embedded redirect to the same URL in the end. 


\section{Chapter 3}

\section{Hadoop and FP-Trees}

This chapter presents the basic concepts of Hadoop and FP-Tree structure that are at the basis of the system presented in this thesis. Section 3.1 presents Hadoop and its basic concepts. Section 3.2 describes what an FP-Tree is and talk about the FP-Growth algorithm. Section 3.3 describes itemsets, what they are, and how they are used.

\subsection{Hadoop}

Hadoop is an open source distributed computing framework. Section 3.1.1 describes the file system used by Hadoop. Section 3.1.2 provides an example that will be used throughtout Section 3.1. Section 3.1.3 explains the input for a Hadoop job. Section 3.1.4 describes the MapReduce framework. Section 3.1.5 describes the Combiner and Paritioner components.

\subsubsection{File System}

The Hadoop Distributed File System (HDFS) is the file system used by Hadoop. It was designed to run on commodity hardware and operate under a set of assumptions. Theses assumptions, outlined in [8], are as follows: 
1. There will be hardware failures.

2. A need to work with large datasets.

3. Spreading computational work is easier than spreading data to where the computation is done.

The most crucial is assumption 3. There are several nuisances with spreading the data to where the computation is done. One such nuisance is space on the machine. The data needed could potentially be larger than the space on the machine. Transfer speed between machines is another nuisance. Datasets are typically larger than the program that will be processing them. So, it is slower to transfer the datasets to where they are needed rather than spreading the program to where the datasets are. The datasets processed in this thesis are between 1 GB to 12 GB each in size, whereas the program that processes them is only $44 \mathrm{MB}$.

The HDFS is setup as a master/slave architecture consisting of a single active NameNode and a number of DataNodes. The NameNode regulates client access to the data. The DataNodes are responsible for managing the storage of datasets, which are stored in blocks. The NameNode tells the client which DataNode to go to access a file. The DataNode then serves the read and write requests. Blocks of data are also replicated across DataNodes. This replication of data attempts to ensure that the Hadoop cluster remains operational despite hardware failures, statisfying assumption 1.

Since data is stored as blocks across DataNodes, it allows for large datasets. This statisfies assumption 2. These blocks also play an important role when a Hadoop job is launched. When a Hadoop job is launched it spawns Map tasks, that are described in Section 3.1.4. How many tasks and on which DataNodes they run are determined by which DataNodes the data that is being accessed resides. If the data resides across five DataNodes, five Map tasks will spawn on those DataNodes. This makes it so there is no need to move data blocks around to complete the job. Instead, the job happens where the data blocks are. These data blocks are also not to be considered as one per a piece of data. Instead, they are considered as as many pieces of data, or even parts of data, that could fit into a block. This reduces the number of Map tasks that spawn during the 
job, which in turn reduces the internal communication that happens, and ultimately statisfying assumption 3 .

\subsubsection{Example Application}

As a running example, let us suppose there is a system that manipulates a large library of books. The system runs Hadoop. This example book system glosses over some of the minor issues, such as books having the same title, to keep things simple.

The book system needs to process all the books and extract usefull information such as:

1. Number of words per book.

2. Number of words per chapter of a book.

3. Number of words per page for a book.

4. Number of chapters per book.

5. Number of pages per chapter for a book.

6. Number of pages per book

7. Number of books for a library.

8. The grand total of each.

Each of the following sections refences this example system.

\subsubsection{Input}

The input of a Map task is typically a file residing on the HDFS, in a format that the MapReduce framework can accept. There are several file formats, most notably the SequenceFile and the MapFile formats. The output could be in the same or different format. 
A SequenceFile is a file consisting of records. Each record holds a key-value pair along with metadata about the size of the record, key, and value. When residing in the file, both the key and value are serialized versions of a Java class that extends class Writable. The header metadata of a SequenceFile contains information about the version of the SequenceFile, class names of the key and value, and compression if used. In order to find a key within it, the SequenceFile is read sequentially until the key is found. This is a drawback when needing to quickly retrieve data based on a given key. In the case of the example book system, the key could be the title of the book. The value could be the content of the book. Each SequenceFile represents a library of books consisting of millions of books.

The MapFile is only slightly more complex while offering direct retrieval of data based on a key. A MapFile is actually a directory that contains two SequenceFiles, that are named the data and index files. The data file consists of the given key-value pairs. The index file consists of only a fraction of the given keys and a byte offset as a value. The byte offset is the position within the data file for that key. The given key-value pairs need to be given in an ascending order when creating a MapFile. When retrieving data for a given key, the index file is loaded into memory and a binary search is performed. The search either finds the exact key and its offset or a key that is near the given one, at which point it goes to the offset and either reads the value or a few more keys down until the one searched for is found within the data file. For the example book system, the key-value pairs would be no different than those used for the SequenceFile. However, when creating the MapFile, the book titles need to be in ascending alphabetical order.

\subsubsection{MapReduce}

MapReduce is a software framework that is used by Hadoop. It is meant to allow processing of large volumes of data in parallel, on a cluster of machines.

A MapReduce job consists of Map and Reduce tasks. Map tasks take data as input, which is chunked across files mentioned in Section 3.1.1. This input is in the form of a key-value pairs. Map 
tasks are ran in parallel, independent of each other on the DataNodes. They contain the data that is used as input. The Map tasks manipulate the key-value pairs and output resulting key-value pairs that are used as input for the Reduce task. The resulting key-value pairs do not need to be of the same type as the ones used for input. Map tasks are composed of setup, map, and cleanup methods. Only the map method needs to be explicitly implemented. The setup method allows set up to be done prior to the map method. The map method is the defining method of the Map task. This is where all the work happens. The cleanup method is ran at the very end, after the Map task is finished processing. It allows to close or finish anything that is necessary.

For the example book system, an input record consists of a key representing a book title and a value representing the contents of the book. The Map tasks counts the number of words per page in each chapter. An output record consists of a key that is the book title and chapter number. The value consists of a page number and the word count for that page. For instance:

("The Gunslinger_2", "1,365")

"The Gunslinger_2" is the key. The value is " 1,365 ". This pair would mean that the book title is "The Gunslinger", it is chapter 2, it is page 1 of the chapter, and the page has 365 words.

The Reduce tasks input are key-value pairs that are produced by the Map tasks. These key-value pairs are different. Each key is associated with a list of all corresponding values as opposed to a singul value. A Reduce task is broken down into three separate phases; Shuffle, Sort, and Reduce phases. The Shuffle and Sort phases happen simultaneously but prior to the Reduce phase. The Shuffle phase fetches the key-value pairs. The Sort phase sorts them based on an implementation of a comparable Java class. The Shuffle and Sort phases ultimately hand off the key paired with a list of all of their associated values to the Reduce phase. In the Reduce phase, the input is further manipulated to produce the desired data. The results are typically written out into a file residing on the HDFS. Similarly to the Map task, the Reduce task is also composed of setup, reduce, and cleanup methods. The reduce method is where all the work is done.

In example book system, the Shuffle phase collects all the keys and the corresponding values, for 
instance:

("The Gunslinger_2", "1,365"), ("The Gunslinger_2", "2,325"), ("The Gunslinger_1", "2,234"), and ("The Gunslinger_1", "1,632")

The Sort phase sorts and hands off the keys and their corresponding values to the Reduce phase, for instance:

("The Gunslinger_1", ["1,632", "2,234"]), and ("The Gunslinger_2", ["1,365", " $2,325 "])$

The values of each key are now lists of corresponding values. In the Reduce phase, the input is further processed to produce required information outlined in Section 3.1.2. The number of values for a given key represents the number of pages for that chapter in that book, which is the 5 th required item. It is then able to count how many chapters there are per book or simply keep the numerically largest one, which is the 4 th required item. The word counts is then summed in various ways to produce words per book, chapter, and page, which are the 1 st, $2 \mathrm{n}$, and $3 r d$ required items respectively. The number of pages per chapter are then summed to produce the number of pages per book, 6th required item. Book titles are put into a set. The size of the set is the number of books in a library. This is the 7 th required items. Further manipulation of these numbers gives the grand total of each for that library, 8th required item.

\subsubsection{Combiner and Partitioner}

The Combiner and Partitioner tasks are auxiliary tasks. They are optional. They happen in-between the Map and Reduce tasks.

The Combiner task is ran right after a Map task has finished. It is only ran if implemented. It takes in the output of a Map task. It runs on the same DataNode as the Map task. Its output is used as an input for the Reduce task. The purpose of a Combiner task is to cut down the amount of data sent to the Reduce task. This is important since the Map and Reduce tasks are typically not ran on the same DataNode. Thus the output of the Map task needs to be communicated to the Reduce task across the network. A Combiner class actually extends the Reducer class. It is 
composed of the same three methods as described for the Reduce task.

Following the example book system and the output of the Map task described in Section 3.1.4, this means that each page of each book in the library is sent to the Reduce task. This causes a lot of communication, since the library contains millions of books and each book contains hundreds of pages. Suppose that the 3rd item, number of words per page, is no longer required. The Combiner can ellimentate that information in the output of the Map task. It does so by doing nearly the same thing as the Reduce task that has been described in Section 3.1.4, that is, it counts the number of words, pages, and chapters per book and number of pages per chapter and book. The output of the Combiner task is a key consisting of the title of a book and name of an item being counted with the value being the count of that item. For instance the record ("The Gunslinger_Chapters", 12), means that there are 12 chapters in the book "The Gunslinger". This means that only a handful of key-value pairs are communicated to the Reduce task as opposed to hundreds of key-value pairs that represent each page of each book in the library, significantly reducing the communication and further processing overhead.

The Partitioner task is always ran prior to the Reduce task, even if not explicitly implemented. The Paritioner takes as input the output of either the Map or Combiner tasks. The input is then partitioned into the number of Reduce tasks. By default a hash function is used to partition the input. The Paritioner controls to which Reduce task the key-value pairs go. This is especially useful in managing congestion caused by keys that are causing heavy computation in the Reduce task or separating data into possibly categories.

The key-value pairs sent by the Map or Combiner tasks of the example book system, split up across Reduce into categories. These categories are book titles that contain the word "the" and book titles that do not contain the word "the". The Partitioner task receives a record and examines the key that contains the title of the book. If it contains "the" in it, then it sends it to the first Reduce task otherwise to the second Reduce task. The output of the first Reduce task then contains the various counts for books that have "the" in the title. The output of the second Reduce task of 
books that do not contain "the" in the title.

\section{$3.2 \quad$ FP-Trees}

Han et al. [1] proposed the Frequent Pattern (FP)-Tree data structure and FP-Growth algorithm. FP-Growth is a data mining algorithm that uses the FP-Tree structure. As the name suggests, the FP-Growth algorithm is about finding frequent patterns within a dataset. It does so by constructing an FP-Tree and then mining it. An FP-Tree is a lossless structure that represents the entire dataset as a hiererchy of items. It is structured such that the items closest to the root are the most occurring ones. Items are relevant parts of an entity, found in the dataset. Each path within the FP-Tree is a representation of a set of items of an entity, re-ordered from the most to the least occurring. When items of multiple entities are added to the FP-Tree, the branching that happens represents the differences between the entities.

Table 3.1: Example entities and itemsets

\begin{tabular}{c|c} 
Entity & Itemset \\
\hline 1 & $\{\mathrm{~A}, \mathrm{~B}, \mathrm{C}, \mathrm{D}\}$ \\
2 & $\{\mathrm{~A}, \mathrm{~B}, \mathrm{C}, \mathrm{D}\}$ \\
3 & $\{\mathrm{~A}, \mathrm{~B}, \mathrm{~K}, \mathrm{~L}\}$ \\
4 & $\{\mathrm{E}, \mathrm{F}, \mathrm{G}, \mathrm{H}\}$ \\
\hline
\end{tabular}

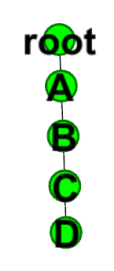

(a) Two identical entities.

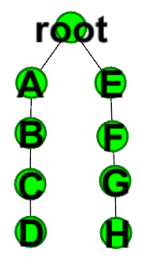

(b) Two different entities.

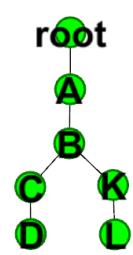

(c) Two similar entities.

Figure 3.1: Example FP-Trees.

Construction of an FP-Tree can be broken down into two parts. The first part reads the set of data and computes a list of items and the number of times they occur. The second part reads the set of data again, constructing ordered itemsets for each entity in the dataset. The itemsets that are created during the second part have their items sorted from the most to the least occuring. Items 
that do not meet a certain occurrence threshold are removed. This threshold is refered to as the minimum support and is determined heuristically. Minimum support reduces the noise that appears on the FP-Tree while keeping the most occuring items. The noise are items that do not occur a significant amount of times. After being created, these itemsets are put into the tree.

As an example, if you take the itemsets of two identical entities, entities 1 and 2 from Table 3.1, and construct an FP-Tree, there is only one branch from the root as shown in Figure 3.1a. Likewise if two completely different entities, 1 and 4, are taken, the FP-Tree has two branches stemming from the root as shown in Figure 3.1b. It is more interesting when the entities share items, such as entities 1 and 3. These differences are seen by having a branch going off on the last shared item of the entities as shown in Figure 3.1c.

\subsection{Itemset}

Recall from Section 3.2 that each path in the FP-Tree represents an entity. Each node is an item of the entity. The items are sorted from the most occurring item closest to the root, to the least occurring item being the leaf. A path is a set of items. In CAFE, the following items are collected from spam emails: character set, content type, encoding, subject, layout, attachment name, MD5 of the attachment, URL path, URL parameter, hostname, and second level domain. Not all of the items may be present in a given spam email.

Figure 3.2 shows two similar emails, with each item identified by a box. The following is the itemset for Figure 3.2a:

$\{($ text/html, content type $),(7$ Bit, encoding $)$, (finewatch2016.ru, second

level domain), (yyyy.finewatch2016.ru, hostname), (E22BKN2RSu4D-nvvfQ9zW7A3tyRw, URL path), (Re: Fantastic selection of brand names, subject), (TTTTTTTTNTTUTTTT, body layout)\}

The following is the itemset for Figure 3.2b: 
Received: from ip-111-bbb-211-133.xxxx.net (HELO 111.bbb.211.133) (111.bbb.211.133)

by n1 (qpsmtpd/0.80) with SMTP; Sun, 07 Aug 2016 23:58:57 +0000 Message-ID: 〈4643437077-HQLKBENOHPFBDSRRAGXEHCX@ $x x x x x x x x . x x>$

From: "Aron Keenan" 〈Keenan60@xxxxx.xxxxx>

Subject: Re: Fantastic selection of brand names subject item

To: $w b-00017 w-00 @ x x x x \cdot x x \cdot x \times x$

Date: Sun, 07 Aug 2016 20:53:53 -0400

Mime-Version: 1.0 content type item

Content-Type: text/html; encoding item Content-Transfer-Encoding: 7Bit $\begin{aligned} & \text { second level URL path item } \\ & \text { domain item }\end{aligned}$

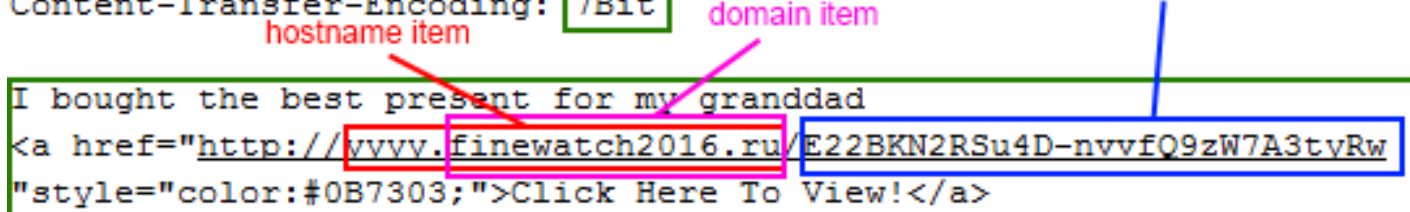

(a) First example spam email.

Received: from ip-121-bbb-211-133.xxxx.net (HELO 121.bbb.211.133) (121.bbb.211.133)

by n1 (qpsmtpd/0.80) with SMTP; Sun, 07 Aug 2016 23:58:59 +0000 Message-ID: <98747342723-AXXDWTLNXZOSBFCCEPMOJIDNT@XXXXX.xxxx.xx> From: "Luciano Evans" <Evans85@xxxxx.xx> Subject: Re: Own a submariner for less than 100 USD today To: $\mathrm{y}-00042 \mathrm{k}-00 \mathrm{exxxx} \cdot \mathrm{xx} \cdot \mathrm{xxx}$ Date: Sun, 07 Aug 2016 22:51:57 -0200

Mime-Version: 1.0 content type item Content-Type: text/html; encoding item Content-Transfer-Encoding: 7Bit second level URL path item Designer branded watches availabje at clearance prices ka href="http:/ xxxx. finewatch2016.rulEdYztQuRDYk-pmK24Kal6GLMNEA "style="color: \#0B7303;">Check New Styles $</ a>$

(b) Second example spam email.

Figure 3.2: Two similar example spam emails.

$\{($ text/html, content type), (7Bit, encoding), (finewatch2016.ru, second level domain), (xxxx.finewatch2016.ru, hostname), (EdYztQuRDYk-pmK24Kal6GLMNEA, URL path), (Re: Own a submariner for less than 100 USD today, subject), (TTTTTTTNTTUTTT, body layout)\} 
produced using the two example emails. Each branch from the root to the leaf represents a spam email. Spam emails that have similar items from the root node down to the last common item split and create two branches, as illustrated in Figure 3.1c. Duplicate spam emails creates a single branch, as explained in Section 3.2 and shown in Figure 3.1a. Different branches are created when two emails do not share common items, illustrated in Figure 3.1b. This creates visually unique trees for each type of botnet, since each of them contains different items.

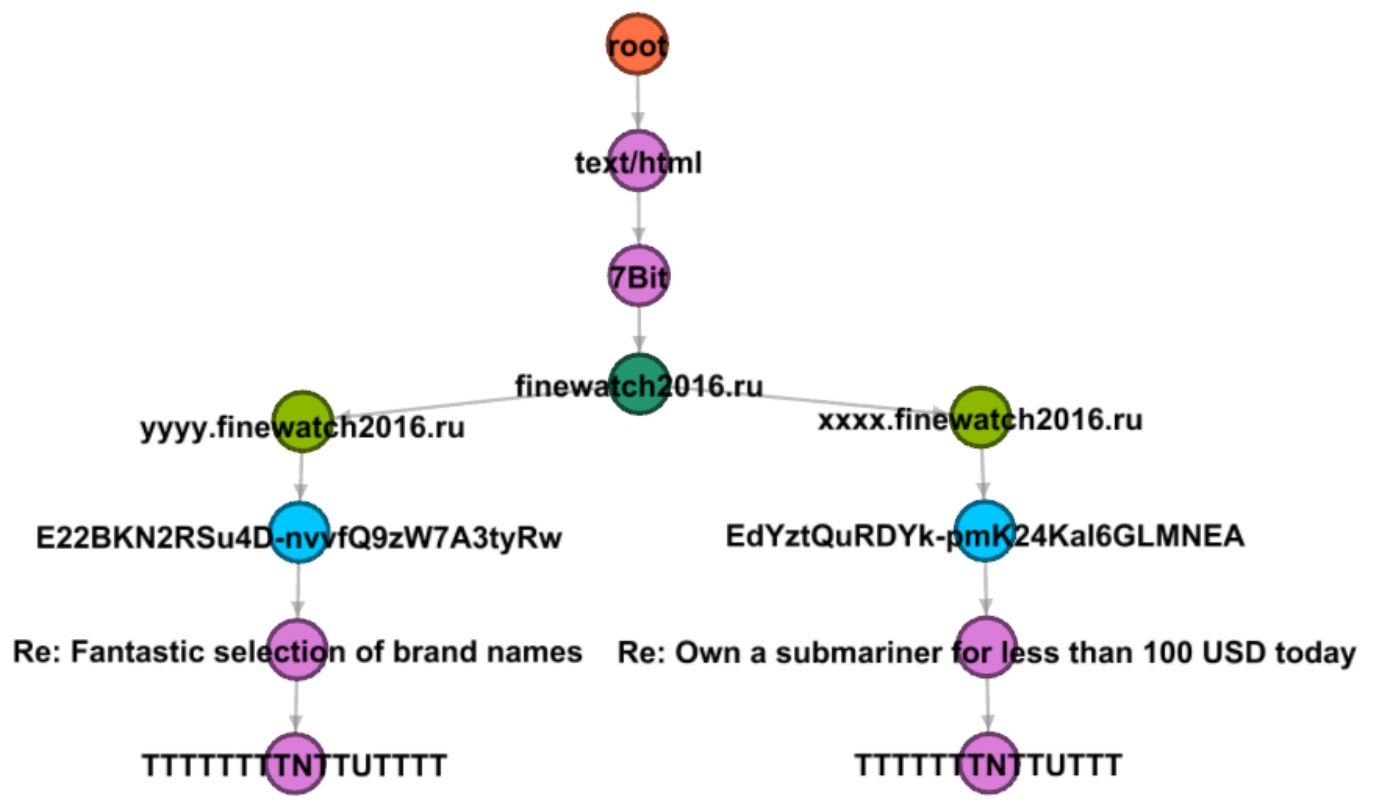

Figure 3.3: Example FP-Tree. 


\section{Chapter 4}

\section{System Design}

The high level architecture of CAFE is shown in Figure 4.1. Each part is described in the following sections. The system starts by receiving spam emails from sources. Which go to the ingestion system that is described in Section 4.1. The spam emails are then processed by the parser, that is described in Section 4.2. Based on the parser's output, a tree is produced by the tree maker, that is described in Section 4.3. Post processing of the parser's output is described in Section 4.4. Optimizations that make the system work better on Hadoop can be found in Section 4.5.

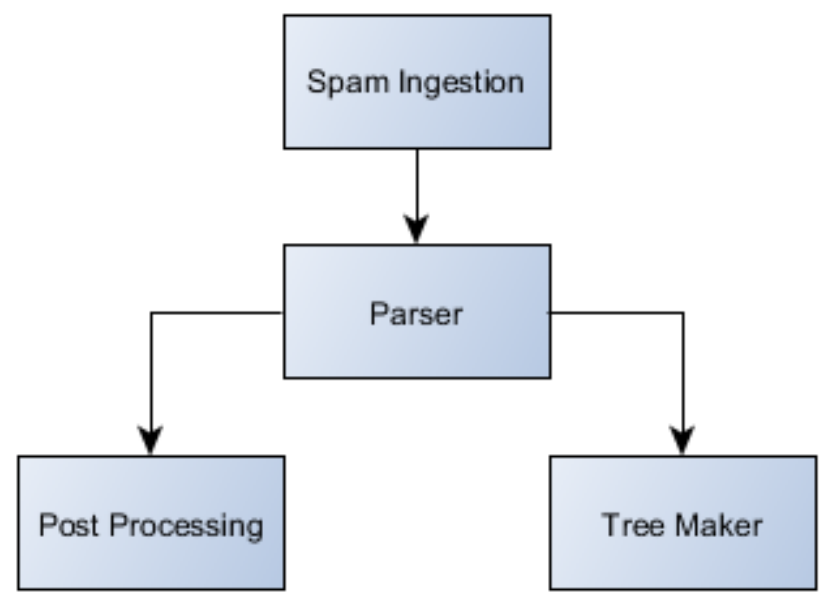

Figure 4.1: System diagram. 
The system is meant to satisfy the following five requirements:

1. Ability to search through spam emails.

2. Ability to retrieve spam emails.

3. Do mass analysis.

4. Store large volumes of spam emails.

5. Track to the origin of spam emails.

Requirements 1, 2, 4, and 5 are satisfied during ingestion, described in Section 4.1. Requirement 3 is addressed in Sections 4.2 and 4.3.

\subsection{Spam Ingestion}

Receiving spam emails in large quantities has its own problems. The problems are:

1. Proper file and folder structure.

2. Not running out of i-nodes and disk space.

3. Creating a format that Hadoop accepts.

4. Being able to retrieve spam emails.

Proper file and folder structure is a problem because it is simply impossible to store millions of files in one folder and be effective, this problem falls in line with requirements 1,2 , and 5 . Running out of i-nodes and disk space is a problem due to the volume of spam that is received. This falls in line with requirement 4. An i-node is a data structure used in Unix style file systems that contains information about a file or a folder. Each file and folder has an i-node. Creating a format that Hadoop accepts is needed to be able to process the spam through Hadoop. This allows for requirement 3 to be 
satisfied, addressed in Sections 4.2 and 4.3. Retrieval of spam emails is important for when detailed analysis is done and the raw spam emails are needed, falling in line with requirements 1,2 , and 5 .

Problem 1, is solved by placing the spam emails into a specific folder structure upon receiving. The folder structure starts with the name of the source then goes into sub-folders by year, month, day, hour, minute, and the last folder being the second on when it was ingested. The name of the file is then made to be a random four digits, which keeps the file names unique within the second folder. This structure is also able to generate spam email IDs that link back to the original email. This also aids with the problem 4, retrieving spam emails. An example spam email ID would be 'src-201605251330010001'. This example spam email ID would create the path '/src/2016/05/25/13/30/01/0001' which leads directly to the spam email. The spam email ID also tells information such has the spam email arriving from 'src' on 2016/05/25 at 13:30:01.

Problem 2, running out of i-nodes and disk space, is solved by having the aforementioned file structure stored into a one large gzipped compressed tar file. The compressed tar file preserves path information. The full path information is also sorted in chronological order inside the tar. The compression reduces the amount of disk space needed. Storing in one file reduces the number of i-nodes used.

Problem 3, creating a format that Hadoop accepts, is solved by reading from the sorted and compressed tar file and writing out into a Hadoop MapFile, described in Section 3.1.1. The MapFile takes in as input a list of keys in an ascending order, which is the unique ID provided through solving problem 1 . The value is the contents of the email.

Problem 4, retrieving spam emails, is solved as follows. The MapFile, as described, takes in a key which is the unique ID of a spam email. It gives back the value, which is the spam email's content. Knowing the unique ID of an email and retrieval of it is done through a function call provided by Hadoop.

Due to problems 2 and 3, the system does not process spam in real time. Instead, all the spam for the day is gathered into the aforementioned large gzipped compressed tar file. At the end of the 
day, the tar file is converted into a MapFile which is then processed. From the time that the first email for a day is received, there is approximately a one day delay to when it is processed.

\subsection{Parser}

The Parser is the most critical component of the system. It and the Tree Maker satisfy requirement 3, mass analysis. The MapFiles generated by the ingestion system, described in Section 4.1, are used as input. Each MapFile contains spam emails from a source on a given day. Segmentation by day is related to problem 1 in Section 4.1. The parser collects primarily information that is then used by other processes to do their task. The Parser itself is a MapReduce job. Its structure is illustrated

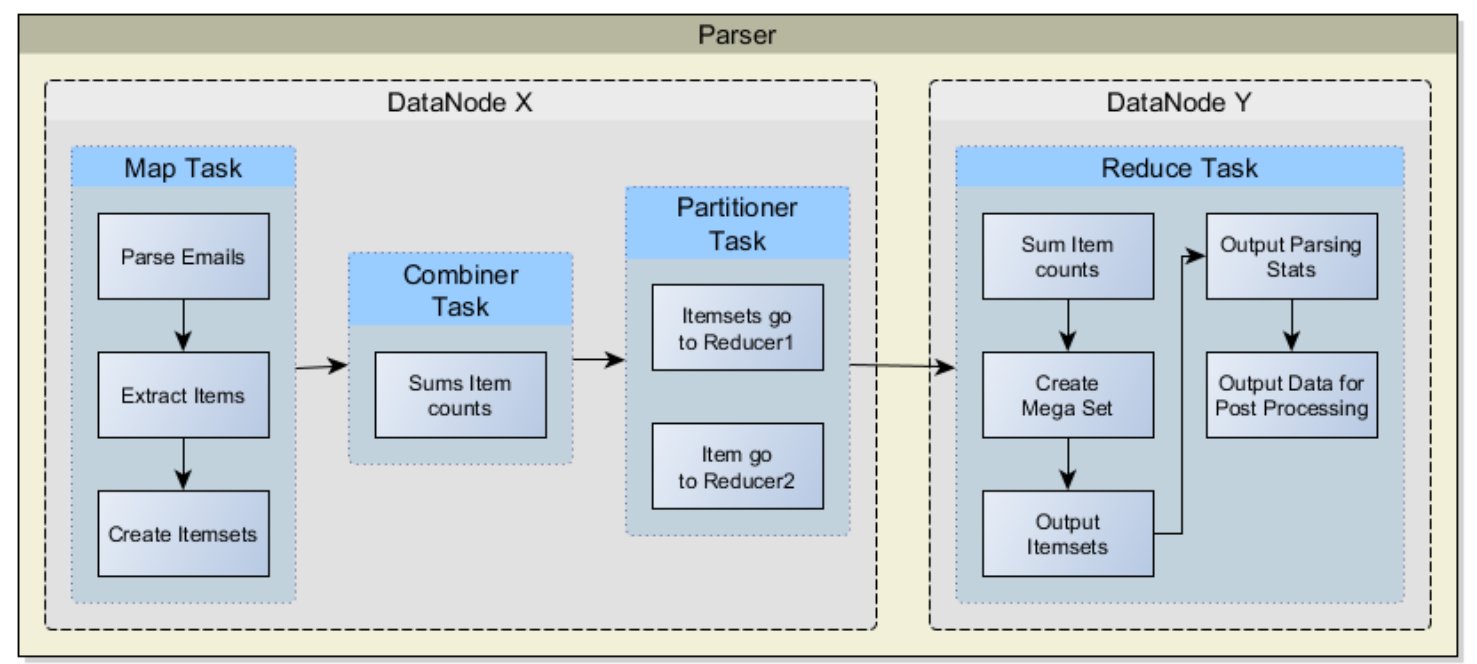

Figure 4.2: Parser Structure

in Figure 4.2. The job can be broken down into the Map, Combiner, Partitioner, and Reduce tasks.

The Combiner and Partitioner tasks are briefly explained here and in more detail in Section 4.5.

They are part of optimization and not a necessity for the system to function. Psuedocode for each task can be found in Appendix A.4.

The Map tasks can be seen as three steps: 1. parsing the spam emails, 2. extracting items, 3. and creating itemsets. Every spam email ID is passed in as a key and corresponding whole spam email 
as the value. The email is parsed as the first step, using a heavily modified email parser. The email parser is developed to handle emails that do not necessarily adhere to standards. The email parser produces an email object, containing items belonging to it. Each item and its type are passed down to the Reduce task, second step, as a key with a numerical value representing the number of occurrences in the email. MD5 of attachment and hostname items are passed down with an email ID as their value. This is to enable tracking and further processing on these specific items. Items from this email object are gathered into an itemset, third step, described in Section 3.3. Itemsets do not hold items in any particular order at this point. The itemset passed down to the Reduce task as a key-value pair. The key is the itemset and value is the email ID. Itemsets may not be unique. Thus the Reduce task may receive an itemset with multiple email IDs as values. The pseudocode for the Map tasks can be found in Appendix A.4 Procedure ParserMap.

Between the Map and Reduce tasks, there are two optional ones, the Combiner and Partitioner tasks. They intercept the key-values that are passed down to the Reduce task and allow for additional work on them before reaching the Reduce task. Both of these tasks run on the same DataNode as the Map task, thus not incurring any transfer cost. The Combiner task is used to combine values for certain keys, for instance summing up all the values for a key before the pairing reaches the Reduce task. The Partitioner task allows for control over which Reduce task a key-value pair will go to. Use of the Combiner and Partitioner tasks is explained in Section 4.5. They are optimizations that could be removed making the Parser function as a simple MapReduce job. The pseudocode for the Combiner and Partitioner tasks can be found in Appendix A.4 Procedure ParserCombine and Procedure ParserPartitioner respectively.

The Reduce task can be broken into five steps: 1 . item values are summed, 2 . unique itemsets are output, 3. statistics are output, 4. an index of items is created, 5 . and items with additional data is output for post processing. Each key seen by the Reduce task is either an item or an itemset. Keys that are an item have the number of occurances in an email as their values. The values are summed providing a total count of an item, as well as a grand total item count. Items are output along with 
their type and total count into an index of items. The index of items is used by the Tree Maker, discussed in Section 4.3. Because keys are unique, each item is only seen once. This allows for a count of unique items. The item count total, as well as unique item count, are output as statistics. Items that need to be tracked or further processed are output into different files that follow a simple format of item and a list of email IDs. If the key is an itemset, with a list of values that are email IDs, then the itemset along with a list of email IDs are written directly to a SequenceFile. The number of email IDs received with the itemsets is counted. The grand total should be equal to the number of emails in the tar archive and MapFile. This ensures that no email is lost along the way. The pseudocode for the Reduce tasks can be found in Appendix A.4 Procedure ParserReduce.

\subsection{Tree Maker}

While the Parser is meant to handle only one day's worth of spam for a given source at a time, the Tree Maker may handle multiple days' worth for several given sources at a time. Being able to pick multiple days to produce an FP-Tree enables one to specify a time span of interest. Having more than one source can potentially reveal overlap between them. It also makes it possible to potentially

label unlabeled spam emails by processing them with labeled spam emails. The Tree Maker is composed of two Hadoop jobs. Figure 4.3 illustrates an overview of the Tree Maker. The purpose of the first job is to merge indexes of items for the given days and sources, filter out items that are not used based on the type, generalize specific items, and to filter out items that do not meet the minimum support threshold. The purpose of the second job is to take in the itemsets generated by the parser, sort the items within each itemset, filter out items based on type and minimum support, construct the FP-Tree, identify clusters, and output the FP-Tree and relevant information. The pseudocode for both of the Hadoop jobs can be found in Appendix A.5.

The first Hadoop job is composed of Map, Combiner, and Reduce tasks. The Map task receives items along with their type and number of occurrences from the indexes of items for the given 


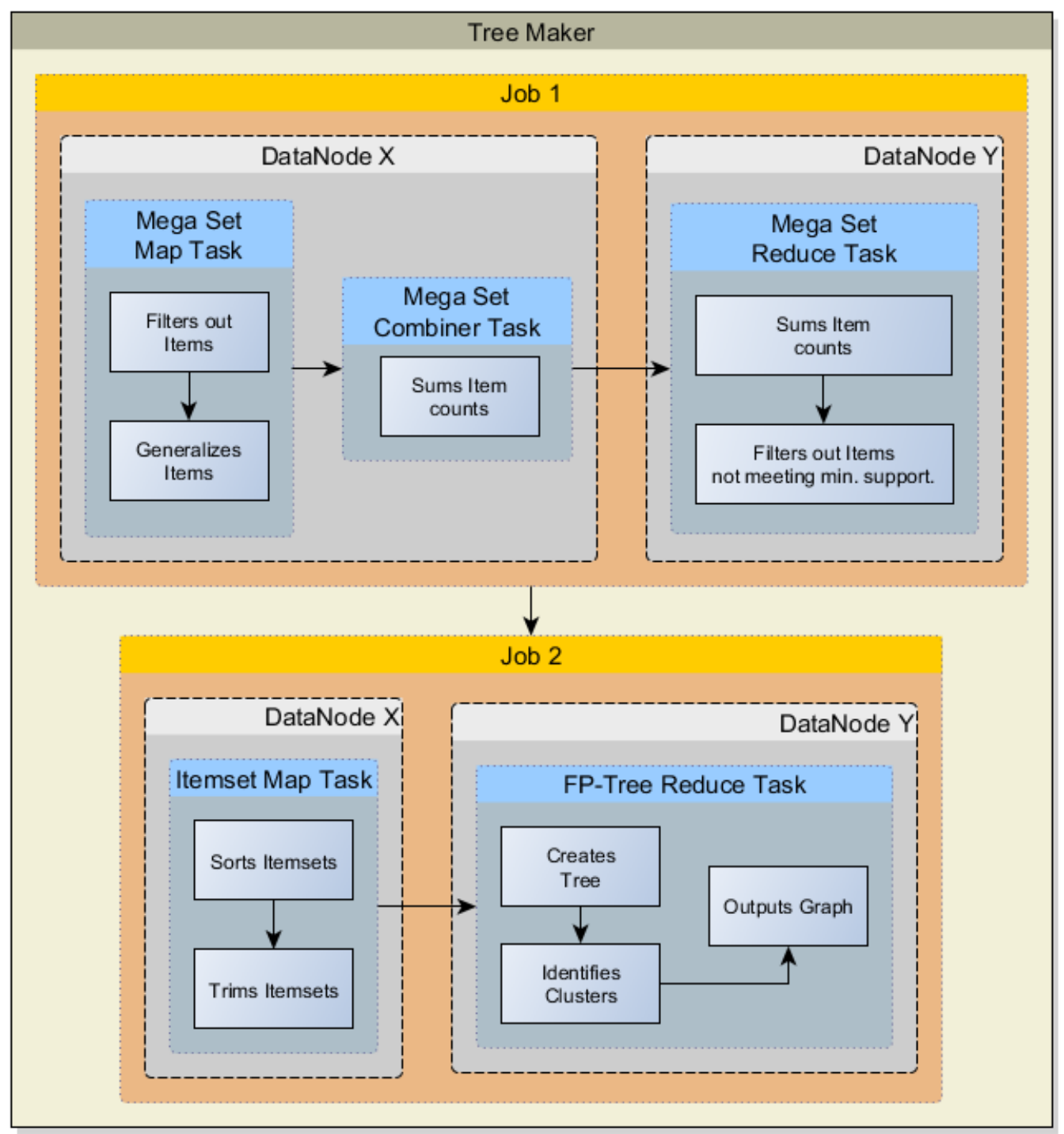

Figure 4.3: Tree Maker overview.

days and sources. The pseudocode can be found in Appendix A.5 Procedure MegaSetMap. Items that have a type found in the list described in Section 3.3 are passed on to the Combiner task unchanged. Items that are either of the type subject or path are generalized before being passed down to the Combiner task. In the case of the subject, the braces, brackets, and parentheses are replaced with a " $\backslash \mathrm{b}$ ", any number is replaced with a " $\backslash \mathrm{d}$ ", and any character found in the

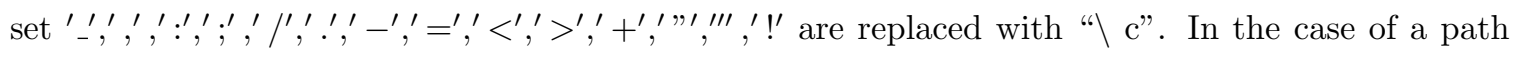




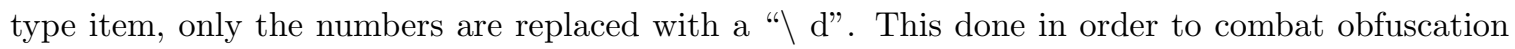
techniques that add random numbers or text emoticons to an item. Items that are not part of the list have no operation done on them and are not passed on to the Combiner task. The Combiner task takes the values of the keys, which are the number of occurrences of an item, and sums them. The pseudocode can be found in Appendix A.5 Procedure MegaSetCombiner. It passes down the same key with only one value to the Reduce task. The Reduce task then performs the final summation per item and rejects any that do not meet the minimum support while outputting those meeting the minimum support into a new index of items. The pseudocode can be found in Appendix A.5 Procedure MegaSetReduce. The minimum support used is determined by:

Let $x$ be the Number of Emails.

Let $\sigma$ be a system parameter.

$$
\operatorname{MinimumSupport}(x)=\left\{\begin{array}{cc}
\sigma x & : x>40000 \\
0 & : x \leq 40000
\end{array}\right.
$$

The resulting item index file spans multiple sources and days, as well as only containing items meeting the minimum support for those sources and days. The system parameter $\sigma$ was set to 0.001125 which was determined after extensive experimentation on datasets containing approximately two million spam emails. For a dataset containing two million emails, the minimum support would be 2250 . This means that any item that does not appear in 2250 spam emails, which is $0.11 \%$ of all of the dataset, is not kept. This greatly reduces the noise created by items that do not appear a significant amount of times.

The second Hadoop job is composed of Map and Reduce tasks. The Map task uses the output from the first Hadoop job as its input. In the setup method of the Map task, the item index is loaded into memory as a map with the key being the item name and the value being the count of the item. The input for the map method of the Map task is the item sets that were produced by the 
Parser. The items within each item set are in ascending order of their count. Each item is checked against the map, that was loaded into memory at setup, to retrieve its count. If the item is not found in the map, it means that it did not meet the minimum support and it is removed from the itemset as part of the trimming. The output of the Map task is then a key that is a sorted and trimmed itemset and a value that is the spam email ID. This allows for a reduction of keys, since multiple emails with different IDs could have the same exact itemset. The pseudocode can be found in Appendix A.5 Procedure TreeMap.

The Reduce task then assembles the FP-Tree. The keys that are received are sorted and trimmed itemsets. Items in the itemsets are taken and inserted into the tree in the order that they are in within the its itemset. The spam email IDs act as a link back to the original spam email and a count of how many times a each itemset has occurred. To insert an item into the FP-Tree, the algorithm checks the current node's, starting at root, children for the same item. If there is a child representing the same item the current node is then set to that child and a counter representing the number of pass through is incremented for that node. The next item from the item set is taken and the same process occurs, creating branches in the FP-Tree. Because each key is unique, the only time that the algorithm goes down the same branch is when an itemset given is a subset or superset of another, thus a shorter or longer version of the same branch. The pseudocode can be found in Appendix A.5 Procedure TreeReduce.

Lastly, in the cleanup method of the Reduce task, the clusters are identified. This happens at the end of the Reduce task, when the FP-Tree had been fully constructed. To detect whether or not a subtree is a cluster, the current node is checked:

1. Current node has not been assigned a cluster number.

2. Current node has a minimum number of children threshold ${ }^{1}$.

3. Current node has an item type that is a cluster legal item type. Based on:

\footnotetext{
${ }^{1}$ This is a system parameter. After expiremenation it was set to 2 .
} 
(a) Item is not "root".

(b) Item is not of type "path" and value of "/".

(c) Item type is not "charset", "contentType", or "encoding".

4. Current node's item's overall occurrence divided by the average occurrence of its children is above a threshold ${ }^{2}$.

5. Current node has a minimum number of leaves threshold ${ }^{3}$.

Each time a cluster is detected a set of all its items is produced and kept track of. This set is then checked for similarity against other itemsets that have been kept, and if a similar itemset is found the current cluster is relabelled to the existing one. Similarity check is done using the Jaccard and Overlap coefficients (see Section 5.1). At the end of detection, further merging is done until no merging is possible. Once no further merging is possible, the tree is output in the GraphML format to be used by visualization software. Itemsets for each cluster is output into seperate files that contain items and their type. The pseudocode can be found in Appendix A.5 Procedure TreeReduceCleanUp and Procedure IdentifyCluster.

\subsection{Post Processing}

After parsing has been completed, additional processes are ran in parallel to each other and the Tree Maker. While these processes do not contribute to making the FP-Tree and identifying clusters, they are used to collect additional information. These processes collect the following things: attachments, IP, and URLs.

Collecting attachments is not done during Parsing because properly containing the attachments and writing to disk are costly operations. Since only the name and MD5 of the attachment are needed for the FP-Tree, the attachment is handled in memory and never written to disk. Instead

\footnotetext{
${ }^{2}$ This is a system parameter. After expiremenation it was set to 1.005 .

${ }^{3}$ This is a system parameter. After expiremenation it was set to 5 .
} 
during parsing, a list of email IDs along with MD5s of attachments that they possess are kept. This list is used by the process that collects the attachments. The process launches a Hadoop job composed of Map, Combiner, Partioner, and Reduce tasks. The Hadoop job goes through the list of email IDs and MD5s created by the Parser and retrieves the attachments from those specific spam emails. The attachments are contained and placed into its output directory. After the Hadoop job is complete, the process retrieves the contained attachments from the output directory and saved them for future processing by external systems.

The Parser also creates a list of last-SMTP-hop IPs along with email IDs that have that IP as their last SMTP server from which the spam email was sent. A process is then ran that geo-locates the IPs and logs metrics into the database. Since it does not contribute to creating the FP-Tree or identifying clusters, while having the cost of communicating with the database, it is done as a separate post-processing process. The results of this post-processing process are then stored in a database for future use by other external systems.

URLs contribute to making the FP-Tree and identifying clusters. However, during parsing and creating the FP-Tree their domains are never resolved. A process goes through all the URLs and resolves their domains as well as geo-locates the resolved IPs, storing all the information in the database for future use by other external systems.

\subsection{Optimizations}

There are four optimizations that were made to improve the performance as well as the dissimilarity of clusters. First optimization is parallelization of FP-Tree creation. Second and third optimizations are implementing an auxiliary Combiner and Partitioner tasks. Fourth optimization is merging clusters.

The first optimization that has been made was the parallelization of FP-Tree creation. Dihn et al. presented Splinter in [2] that also creates an FP-Tree for the same purpose. They have used the 
FP-Tree algorithm in a standard non-parallelized way. The FP-Tree algorithm can be broken down into two parts, as described in Section 3.2. The two parts can be split apart. As presented in this thesis, the construction of the FP-Tree can be broken down into two main Hadoop jobs. The first being the Parser, mentioned in Section 4.2, that does the first part of creating an FP-Tree. The Parser job counts how many times an item has occurred. It also produces the unsorted itemsets for each email that is later used by the Tree Maker described in Section 4.3. Hadoop excels at counting and is being utilized as such by the Parser. The Tree Maker then receives a list of items, for each of them, the number of times it has occurred and a set of unsorted itemsets made by the Parser. Sorting of individual itemsets can happen in parallel to one another. And because the Parser and the Tree Maker are two separate jobs, the Parser can move on to parse the next set of spam emails while the Tree Maker creates the tree for the current set of spam emails.

The next optimization that was made is introducing the Combiner task, between the Map and Reduce tasks for the Parser and Tree Maker, when creating the unified item index. Because the Map and Reduce tasks happen on different machines the Combiner reduces the network communication between the Map and Reduce tasks as well as reducing some of the computational workload that will need to be done by the Reduce task. Both of these things contribute to an increase in performance.

The Paritioner task also helped to optimize the overall performance. While it is possible to have multiple Reduce tasks just by specifying in the Hadoop's job configuration, a Partitioner task has to be written in order to control to which Reduce task a key goes. By default when there are multiple Reduce tasks the destination of a key is determined by its hash value, Reduce tasks are essentially assigned a hash range that are sent to them. Controlling which key goes to which Reduce task allows to manage workloads between the Reduce tasks. For the Parser, keys that are sent to the Reduce tasks fall into three groups. The first group of keys are itemsets. Despite itemsets being keys, there is not a significant amount of identical itemsets. This is because items within those itemsets could potentially be one character different. Because itemsets are relatively unique, this means that one of the Reduce tasks receive roughly the same amount of itemsets as there are emails. Itemsets are 
also large and need to be written out to a file. As such itemsets are sent to one specific Reduce task that handles them. The second group are $\mathrm{JSON}^{4}$ objects of emails. They are once again unique and larger than itemsets. The purpose of them is to be used by another system. They are also sent to another specific Reduce task to be handled solely by it. The last are all the individual items and their counts. The Reduce task assigned to handle them needs to sum the count of each item as well as the grand total, provide statistics on how many of each item and type and output it into a file.

Cluster merging is another optimization that was made to improve the dissimilarity of cluster. It was observed that without merging, there are more clusters. Some of the clusters have items that are seen in other clusters, meaning that they are part of the same cluster. To overcome this, after detection, clusters are merged together based on how similar they are. The full process is described in Section 4.3. To evaluate the results, the Silhouette coefficient is used to score each cluster. Details on the evaluation methodology can be found in Chapter 5. Results can be found in Chapter 6. In general, merging clusters improves the Silhouette coefficient per cluster, thus showing that with merging it is able to produce more dissimilar clusters.

\footnotetext{
${ }^{4}$ JavaScript Object Notation. This notation is not restricted to JavaScript use. It is a commonly used data exchange language.
} 


\section{Chapter 5}

\section{Evaluation}

This chapter outlines the methodology that is used to evaluate CAFE. It presents the way that clusters are assessed for similarity. Section 5.1 explains the metrics that are used to assses similarity. Section 5.2 presents the methodology used to evaluate the clusters. Section 5.3 describes the platform that CAFE is using.

\section{$5.1 \quad$ Metrics}

Early implementations, based on Splinter's, of the cluster construction algorithm showed that some of the clusters were practically identical to others. This is due to excluding some item types from being the start type of a cluster. For instance, if two practically identical spam emails had only different character sets, that appear at the top of the tree, then they are not identified to the same cluster despite having all the other items identical. To overcome this, upon formation of a cluster, the set of all items is checked for similarity against other already assembled clusters. If a certain score threshold is met, then the newly detected cluster is indentified to the old one. Jaccard and Overlap coefficients are used for merging, this is described in Section 5.1.1. Evaluation of intracluster similarity and inter-cluster dissimilarity is done using Silhouette coefficients, explained in 
Section 5.1.2.

\subsubsection{Jaccard and Overlap coefficients}

In CAFE, the Jaccard [9] and Overlap [10] coefficients are used to score the degree of similarity between two clusters.

Let $A$ and $B$ be two sets. The formula for the Jaccard coefficient is as follows:

$$
J(A, B)=\frac{|A \cap B|}{|A \cup B|}
$$

The formula for the Overlap coefficient is as follows:

$$
O(A, B)=\frac{|A \cap B|}{\min (|A|,|B|)}
$$

In the case of spam, the sets A and B represent all items from two clusters. Higher coefficients indicate that there are more items in common between the two clusters. The Jaccard coefficient favors two sets having the same or nearly the same size. When intersection between a set and a small subset of it is taken, the result is smaller than their union. This is a problem. The Overlap coefficient on the other hand handles the possibility that one of the sets is a subset of the other.

Both Jaccard and Overlap coefficients are done on sets of items and do not account for the finer difference between items such as upper and lower case differences. These two metrics are used during cluster merging, which is explained in Section 4.3. During cluster merging we look for clusters that share the same items but have been identified as different clusters. This is possible because the difference between the two clusters can happen very close to the root on an item type such as the character set. This would cause the two clusters to be in two seperate sub-trees while having the same items as leaves. 


\subsubsection{Silhouette coefficient}

To check for intra-cluster similarity and inter-cluster dissimilarity, Silhouette coefficients [11] are computed between each cluster. Similarity is computed by various algorithms. It can be described as how close two items are to one another, in terms of having the same exact characters. Similiarity is given on a 0 to 1 scale of real numbers. Dissimilarity is how distant are two items from being same, in terms of having the exact same characters. To compute dissimilarity the similiarity of the two items is subtracted from 1. The formula for the Silhouette coefficient is as follows:

Let $i$ be an item in a cluster.

Let $a(i)$ be the average dissimilarity between $i$ and all the other items in the cluster.

Let $b(i)$ be the lowest average dissimilarity between $i$ and all the other items in other clusters.

$$
S(i)=\frac{b(i)-a(i)}{\max (a(i), b(i))}
$$

The value of the Silhouette coefficient ranges from -1 to 1 . The higher the value the more similar the item is to items in its own cluster and not to items in other clusters. The lower the value, the more similar it is to items in other clusters as opposed to items in its own cluster. The mean for all the items in a cluster is the Silhouette coefficient for that cluster. Unlike when merging clusters, the item type matters when computing the silhouette coefficient. Item types such as body layout and character set can never match, thus skewing the results. Each cluster itemset is divided into subsets based on item types.

When computing the Silhouette coefficient, individual items are checked for their dissimilarity. The type of check that is done on an item depends on its type. If the item type is either a character set, MD5 of an attachment, content type, or encoding, then the item values are checked for equality. These types score either 0 , if they are different, or 1 , if they are the same.

If the type is the layout of the message body, described in Section 2.2, then the string similarity of the two layout items is computed and multiplied by the string similarity of the base form of 
the layouts. For instance, the string similarity of 'TTTTTUNTTTTT' and 'TTTTTUNTTNTT' is computed and multiplied by the string similarity of their base form, which is 'TUNT' and 'TUNTNT' respectively. This is done due to the limited character set of layouts having a negative impact on string similarity. The multiplication of the string similarity of compressed forms is done to emphasize the difference between two layouts that do not share a potential base layout. It was observed that some spam campaigns paraphrase sentences in their messages. However, the overall structure is the same.

All other types of items are compared by their string similarity. The string similarity is computed as the average of the Levenshtein distance [12], Needleman Wunch algorithm [13], and Jaro Winkler distance [14]. These alogrithms were chosen as they complement each other. Jaro Winkler produces better scores when there is loss of words, small changes such as a missing character, difference in punctuation, and spacing. However, Jaro Winkler produces worse results when words are rearranged. Needleman Wunch and Levenshtein can handle rearrangement of words, thus they make up for Jaro Winkler. Levenshtein is case sensitive and produces worse results when the casing does not match, Jaro Winkler and Needleman Wunch make up for it. The SimMetrics library, open source and developed by Sheffield University, is used in the implementation of CAFE.

\subsection{Methodology}

Once the Tree Maker, described in Section 4.3, has finished running it produces a file in the GraphML format and a file per cluster containing its itemset. The file containing the itemset of clusters is used to evaluate clusters. These itemsets are evaluated using the Silhouette coefficient, explained in Section 5.1, to show the intra-cluster similarity and inter-cluster dissimilarity. This produces one Silhouette coefficient per cluster for a given dataset. The overall Silhouette coefficient for a dataset is computed as the average Silhouette coefficient of all the clusters for the dataset. This is done to show the overall impact of applying cluster merging on datasets. Datasets that do not have 
their clusters merged, have low Silhouette coefficients as opposed to datasets that have their clusters merged. Their Silhouette coefficients per cluster are lower since there are clusters that are similar to each other.

Each dataset represents a day worth of emails for a source that provides spam. The Tree Maker produces an FP-Tree with clusters merged and with clusters not merged for each day and source. This allows us to compare the number of clusters as well as their Silhouette coefficients for a source against multiple days. While it is possible to produce FP-Trees that span multiple days or multiple sources, this has not been done during experimentation. Creating FP-Trees that span multiple sources per day would create FP-Trees that show common items, thus patterns, among the sources. FP-Trees that span multiple days per source would show items that persist for a source through time. Combination of the two shows common items among sources that persist through time.

\subsection{Platform}

The platform that is used is the Hortonworks Data Platform (HDP). HDP provides a software stack that runs a Hadoop cluster of processors, as well as additional services that manage the cluster or augment Hadoop's capabilities. Cluster management services are used, but the additional services that augment Hadoop's capabilities are not used. More specifically, only the following are used by CAFE: HDFS, MapReduce, YARN, and common utilities that support those services. HDFS and MapReduce had been discussed in Section 3.1. YARN is a framework for cluster resource management and job scheduling. We use YARN to launch jobs. YARN decides when to start a job, dependent on having enough free resources available. YARN also dictates exactly how much RAM is allocated on each machine, for each MapReduce job, and for each task in a MapReduce job.

CAFE is responsible to launch the processes described in Chapter 4. Every process that utilizes Hadoop is launched using YARN. CAFE is also used to run the Splinter system. When running Splinter on CAFE, all other processes are stopped. Only those needed by Splinter are running. 
CAFE also runs the process that evaluates clusters.

Table 5.1: Machine types and CPU models.

\begin{tabular}{|c|c|c|c|c|c|}
\hline Type & CPU Model & Clock Speed & CPU cores & Threads & Machines \\
\hline VM & $\begin{array}{c}\text { Intel(R) Xeon(R) } \\
\text { CPU E5-2620 }\end{array}$ & $2.00 \mathrm{GHz}$ & 4 & 4 & CAFE \\
\hline VM & $\begin{array}{c}\text { Intel }(\mathrm{R}) \text { Xeon }(\mathrm{R}) \\
\text { CPU E5-2620 }\end{array}$ & $2.00 \mathrm{GHz}$ & 8 & 8 & $\begin{array}{l}\operatorname{dn} 1, \operatorname{dn} 2, \operatorname{dn} 3 \\
\text { dn } 4, \text { esn, esn } 2 \\
\text { nn, snn }\end{array}$ \\
\hline Physical & $\begin{array}{l}\text { Intel(R) Core(TM) } \\
2 \text { Duo CPU E8400 }\end{array}$ & $3.00 \mathrm{GHz}$ & 2 & 2 & $\begin{array}{l}\operatorname{dn} 20, \operatorname{dn} 25, \operatorname{dn} 26 \\
\operatorname{dn} 27, \operatorname{dn} 28\end{array}$ \\
\hline Physical & $\begin{array}{c}\text { Intel(R) Core(TM) } \\
2 \text { CPU } 6600\end{array}$ & $2.40 \mathrm{GHz}$ & 2 & 2 & $\operatorname{dn} 21$ \\
\hline Physical & $\begin{array}{c}\text { Intel(R) Core(TM) } \\
2 \text { Quad CPU Q6600 }\end{array}$ & $2.40 \mathrm{GHz}$ & 4 & 4 & $\operatorname{dn} 22, \operatorname{dn} 23$ \\
\hline Physical & $\begin{array}{l}\text { Intel }(\mathrm{R}) \text { Xeon }(\mathrm{R}) \\
\text { CPU W3580 }\end{array}$ & $3.33 \mathrm{GHz}$ & 4 & 8 & $\begin{array}{c}\operatorname{dn} 5, \operatorname{dn} 6, \operatorname{dn} 7, \\
\operatorname{dn} 8, \operatorname{dn} 9, \operatorname{dn} 10, \\
\operatorname{dn} 11, \operatorname{dn} 12, \operatorname{dn} 14, \\
\operatorname{dn} 15, \operatorname{dn} 16, \operatorname{dn} 17, \\
\operatorname{dn} 18, \operatorname{dn} 19\end{array}$ \\
\hline
\end{tabular}

Table 5.2: Disk space and RAM of machines.

\begin{tabular}{cccc} 
RAM & Total Disk Space & Number of Disks & Machines \\
\hline $15.67 \mathrm{~GB}$ & $70.00 \mathrm{~GB}$ & 1 & CAFE \\
\hline $30.37 \mathrm{~GB}$ & $5.00 \mathrm{~TB}$ & 1 & $\begin{array}{c}\mathrm{dn} 1, \mathrm{dn} 2, \\
\mathrm{dn} 3, \mathrm{dn} 4\end{array}$ \\
\hline & & 5 & $\begin{array}{c}\mathrm{dn} 5, \mathrm{dn} 6, \mathrm{dn} 7, \\
\mathrm{dn} 8, \mathrm{dn} 9, \mathrm{dn} 10, \\
\mathrm{dn} 11, \mathrm{dn} 12, \mathrm{dn} 14, \\
\mathrm{dn} 15, \mathrm{dn} 16, \mathrm{dn} 17, \\
\mathrm{dn} 18, \mathrm{dn} 19\end{array}$ \\
\hline $7.53 \mathrm{~GB}$ & $3.63 \mathrm{~TB}$ & 4 & $\mathrm{dn} 20$ \\
\hline $6.70 \mathrm{~GB}$ & $6.38 \mathrm{~TB}$ & 7 & $\mathrm{dn} 21$ \\
\hline $7.62 \mathrm{~GB}$ & $5.46 \mathrm{~TB}$ & 6 & $\mathrm{dn} 22, \mathrm{dn} 23$ \\
\hline $7.53 \mathrm{~GB}$ & $3.63 \mathrm{~TB}$ & 4 & $\begin{array}{c}\mathrm{dn} 25, \mathrm{dn} 26, \\
\mathrm{dn} 27, \mathrm{dn} 28\end{array}$ \\
\hline $7.69 \mathrm{~GB}$ & $50.00 \mathrm{~GB}$ & 1 & $\mathrm{esn}$ \\
\hline $7.69 \mathrm{~GB}$ & $48.00 \mathrm{~GB}$ & 1 & $\mathrm{esn} 2$ \\
\hline $15.58 \mathrm{~GB}$ & $99.00 \mathrm{~GB}$ & 1 & $\mathrm{nn}$ \\
\hline $7.69 \mathrm{~GB}$ & $56.00 \mathrm{~GB}$ & 1 & $\mathrm{snn}$ \\
\hline
\end{tabular}

When a Hadoop job is running, the DataNodes are doing the work of the job. DataNodes are named dn1 through dn28. Name Node (nn) manages DataNodes. The Secondary Name Node (snn) is on standby as replacement to the nn, should it goes down. Extra Service Node (esn) and esn2, run additional services that augment Hadoop's capabilities. 
A mixture of virtual and physical machines have been used, denoted as VM and Physical. CPU details as well as whether a machine is a vritual one or a physical one can be found in Table 5.1. The RAM and storage of the machines can be found in Table 5.2.

CAFE is architecturally different from Splinter, which is described in Section 2.2. Splinter is composed of multiple Python scripts. One script parses the emails and stores the parsed data into an OrientDB. Another script is ran after the first to detect clusters. Additional scripts can be ran to provide other information. Each of the scripts are built to run manually when needed and run on a single machine, process, and thread. In contrast, CAFE is written in Java. There are several processes running autonomously and in parallel to one another. Each is described in Chapter 4. The ingestion is the only process that runs on a single machine and thread. The Parser, TreeMaker, and other post-processing processes utilize the Hadoop cluster. One commonality between Splinter and CAFE is how the FP-Tree is built. Nodes are inserted in a similar manner. Detection of clusters is also similar between the two systems, with the key difference being that CAFE merges clusters as it detects them. CAFE also does merging after clusters have been detected, until no merging is possible. 


\section{Chapter 6}

\section{Results}

This chapter presents the results of various experiments. Section 6.1 describes the datasets. Section 6.2 presents data about clustering. Section 6.3 shows the performance of the system.

\subsection{Datasets}

The grand total dataset contains approximately two billion spam emails, averaging two million spam emails per day for the past three years. A portion of the sources provide spam emails that have been attributed to a botnet. The attributed botnets are: Cutwail, Darkmailer, Gamut, Kelihos, Lethic, Sendsafe, and Slenfbot. Botnets do not send only one type of spam emails. Each botnet has possibly multiple types that could potentially be attributed to spam campaigns. The botnets that have been picked were all the botnets that we had access to. Each botnet has different characteristics from other botnets and produces differently structured FP-Tree. This allowed the system to be tested more throughly. The dates of datasets had been picked at random.

Cutwail has three types of emails. The first type is an attempt to get the victim to check their account and provide a malicious URL link. The second type is a HTML message body about casino deals, containing malicious URL links. These URLs are shortened. The third type is hooking up 


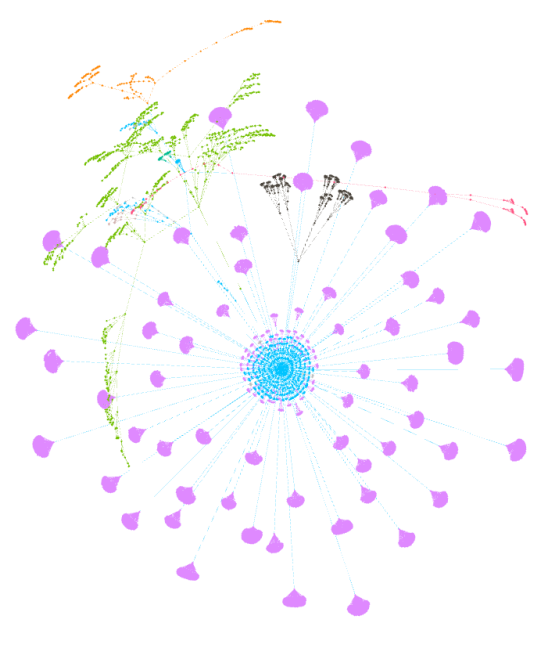

(a) Cutwail.

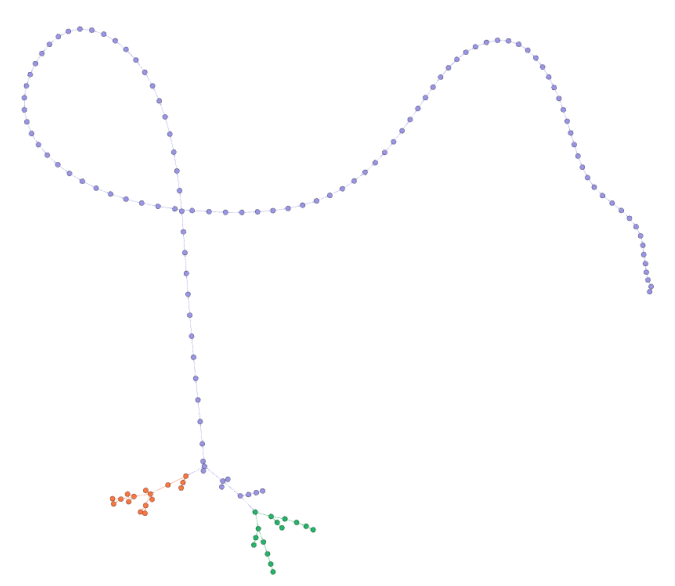

(b) Darkmailer.

Figure 6.1: Cutwail and Darkmailer FP-Trees.

advertising, attempting to get the victim to click on a malicious URL link. There is only a handful of domains that the URL points to. After a couple of days, the domains are switched to different ones. Figure 6.1a is an FP-Tree generated from the Cutwail spam. Different colors indentify different clusters within the graph, light blue nodes have not been clustered.

Darkmailer started with two types in 2015 and changed into three types in 2016. The first of type of 2015 is a base64 encoded HTML message offering to produce oil paintings of photos that are sent to them. The second type of 2015 is hooking up advertisement, there is no nationality of women. It is also a multipart message. It contains a plain text version of itself and an HTML version. In 2016, three types emerged. The first is similar to the first one from 2015. It is a base64 encoded HTML message with a catalogue of oil paintings for purchase. Each image in the catalogue is linked from a URL. The URLs contain only one domains and very long patterned URL paths that lead to the image. The second of the three types is again a hooking up advertising, also multipart message. The nationality of women in the advertising is Russian. The multipart message contains a plain text and HTML version of the hookup advertisement in English. Figure 6.1b is an FP-Tree generated from Darkmailer spam. The long branch of nodes in purple is the oil painting type that started in 2016, most of the nodes being URL paths. The green nodes are the second type, hooking 
up advertisement, that started in 2016. The FP-Tree makes the different types within Darkmailer visually clear. A long branch of nodes indicates that there are spam emails with a lot of items. This is a very distinct feature of Darkmailer. Furthermore, having no other branches splitting off from the long branch means that all of those emails that are on that branch have exactly the large number of items that are the same.

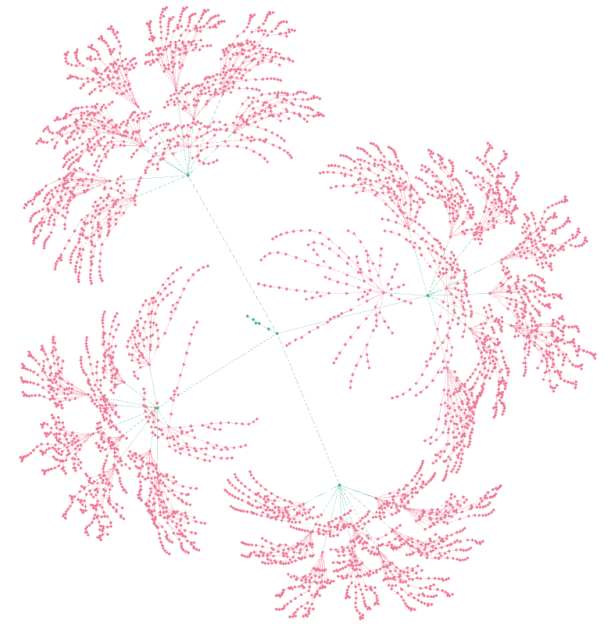

(a) Gamut.

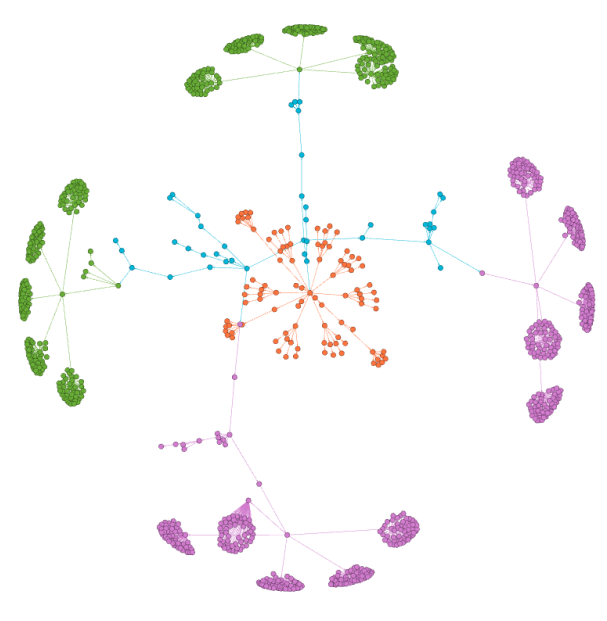

(b) Kelihos.

Figure 6.2: Gamut and Kelihos FP-Trees.

Gamut has four types of emails. The first type is hooking up advertisement. The subject contains the user name of the address that was sent to and a phone number to either call or text. The message contains an HTML encoded message. The encoding causes obfuscation of URLs that are present in the message. The second is also a hookup advertisement. It does not contain the user name nor a phone number in the subject. It is multipart and contains a plain text version of itself and an HTML version. The HTML versions contains a URL. It is not encoded nor obfuscated in any way. The third type is a plain text message about an invoice or a payment and contains a zip file attachment. Type four is job offerings to make large amounts of money. It is multipart with plain text and HTML versions. The HTML is not encoded nor obfuscated and contains a URL link. Figure 6.2a is an FP-Tree generated from the Gamut spam. The green color represents nodes that have not been clustered while the red repesents one cluster. Inspecting the graph for that day shows that there 
was only one campaing about dating. Because all the clustered nodes are of types hostname, URL, and URL parameter, it is hard to distinguish if it is the first or the second type of Gamut, though both them are about dating.

Kelihos has three types. The first type is either about invoice, inventory report, bill, or item list. All of them contain a zip attachment. The second type is an advertisement for a manager course. It is base64 encoded. It contains hundreds of lines of text about the course and registration procedure. The end of the message contains contact information: names, an email address, and phone numbers that are always the same. The third type has an HTML body that has a table. The table has an unsubscribe link and an image tag that references an attached image file. The image tag is linked to a malicious website. Figure $6.2 \mathrm{~b}$ depicts an FP-Tree generated from the Kelihos spam emails. The green and purple nodes represent the third type the HTML message with an image. The orange nodes represent the first type, invoices with attachments. Some light blue nodes represent the second type, about manager course. Once again, the FP-Tree makes it easy to see that there are different types of spam emails produced by the same botnet.

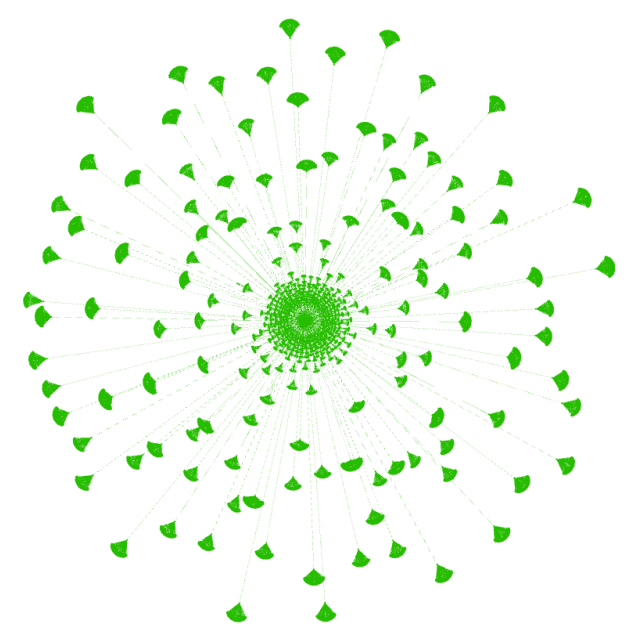

(a) Lethic.

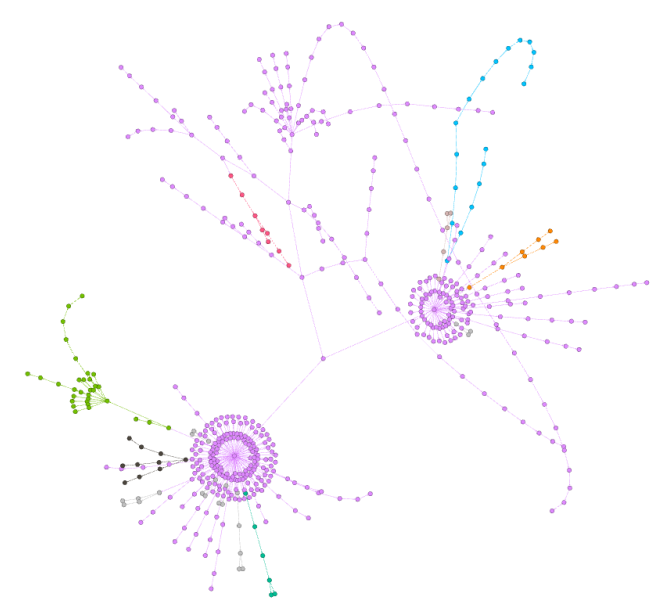

(b) Sendsafe.

Figure 6.3: Lethic and Sendsafe FP-Trees.

Lethic has one type of messages. The message are in HTML and are about selling luxury items such as watches or bags. Each contains one line of text describing what is being sold, and a link. 
The link started as Google redirects, but after a while it switched to its own URL. It aways has two parameters, a $s a$ parameter with a constant value of $D$ and a $u s g$ parameter with a value that resembles a base64 encoded string. Figure $6.3 \mathrm{a}$ is an FP-Tree generated from the Lethic spam. It is considered as one cluster, represented by one color. The center point being Google's hostname, which is used for redirection.

Sendsafe has four types. All the types are directed towards having the victim send private financial information. The first type is a Nigerian money scam. The message format varies between plain text and HTML. The second type is a investment scam. The message format is in HTML. The third type is an FBI scam. The message format is in plain text. The fourth type is a lottery scam. The message format is plain text. Figure 6.3b depicts an FP-Tree generated from Sendsafe spam emails. The short paths represent the first and third types. The longer branches of nodes represent the fourth type. The very long branches represent the second type.

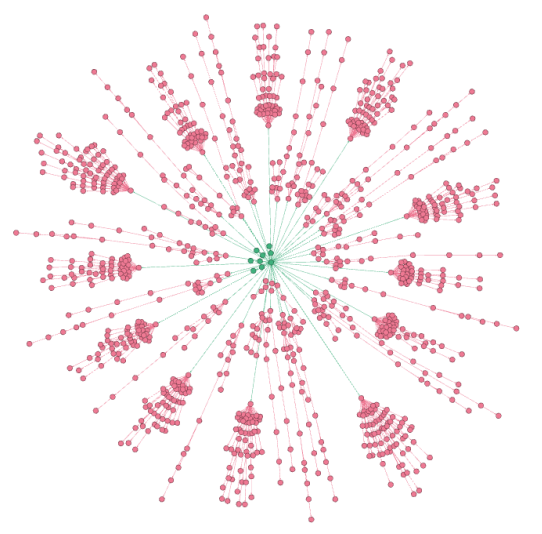

Figure 6.4: Slenfbot.

Slenfbot has one type of emails. The message is in plain text, contains one line that has a sexual context and one URL. Figure 6.4 depicts an FP-Tree generated using Slenfbot spam emails. All the nodes belong to the same type are colored red. Green represents nodes that are not part of a cluster. The center of the graph contains the root node, a "text/plain" content type node, and a "/" URL path node. The red nodes closest to the center are either layouts or second level domains that are from the URLs. 
Lastly, there is a dataset containing spam emails that have not been attributed to a botnet. This dataset is refered to as Unknown. These spam emails may or may not be distributed by the botnets that have been mentioned. They may or may not also be distributed by botnets that have not been mentioned. They may also be test runs of botnets, or very specific and target campaigns. To reinstate, they are not attributed to a botnet, and are considered Unknown.

\subsection{Clustering}

The original algorithm for clustering was presented by Dihn et al. [2] for the system called Splinter. The algorithm had been rewritten and modified to be able to run on and take full advantage of Hadoop, granting an increase in performance and scalability. It was further improved to cluster better by detecting and merging similar clusters prior to completion. Original Splinter code was also used to compare against.

Table 6.1: Datasets used by both systems.

\begin{tabular}{ccc} 
Date & Name & Number of Emails \\
\hline 20160511 & Cutwail_1 & 103571 \\
20160424 & Cutwail_2 & 101309 \\
20160605 & Darkmailer_1 & 67403 \\
20160515 & Darkmailer_2 & 61102 \\
20160317 & Gamut_1 & 107129 \\
20160214 & Gamut_2 & 107730 \\
20160406 & Kelihos_1 & 100878 \\
20160513 & Kelihos_2 & 74212 \\
20160226 & Lethic_1 & 107470 \\
20160103 & Lethic_2 & 99274 \\
20160514 & Sendsafe_1 & 32507 \\
20160511 & Sendsafe_2 & 49686 \\
20160606 & Slenfbot_1 & 62529 \\
20151205 & Slenfbot_2 & 48460 \\
20160505 & Snowshoe_1 & 3325 \\
20160513 & Snowshoe_2 & 3278 \\
20160329 & Spamsalot_1 & 1895 \\
20160324 & Spamsalot_2 & 2403 \\
\hline
\end{tabular}

Table 6.1 presents the datasets used, date that the dataset was collected, name of the dataset, and number of emails in the dataset. When picking these datasets, the limitation of Splinter was 
considered and the number of emails was kept at less than 110,000 emails. For each botnet two datasets have been picked. Each one representing an arbitrary date. These datasets have been picked out of 4602 other datasets at the time of this writing. Figure 6.5 presents the number of

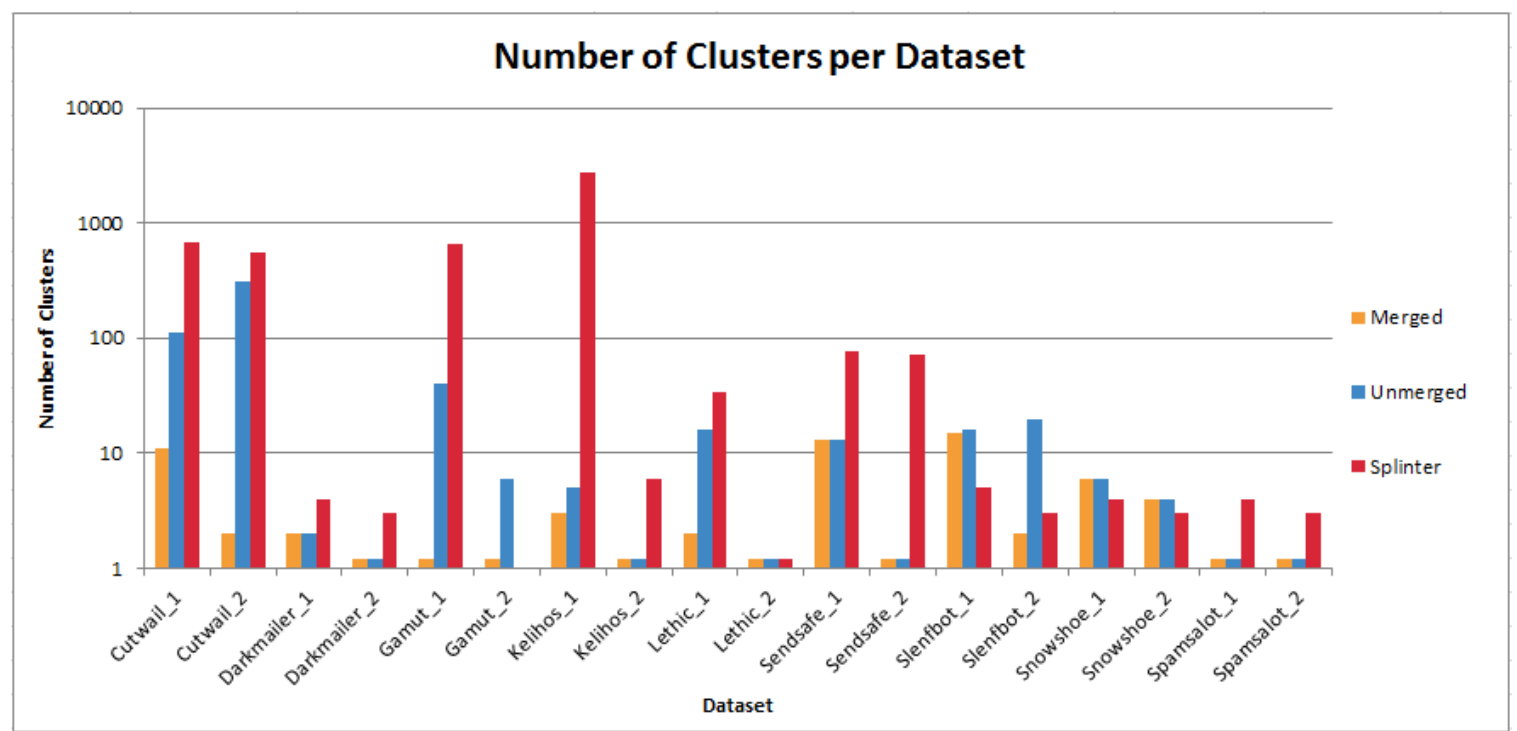

Figure 6.5: Number of clusters produced by each system for each dataset.

clusters detected for the given datasets. For each dataset there are three cases: number of clusters detected by original Splinter and by CAFE with merging, and without merging similar clusters. In most cases, there are less clusters produced by CAFE that are more dissimilar relative to each other. The table containing the full data used to create Figure 6.5 can be found in Appendix A.2 Table A.3.

Figure 6.6 presents the Silhouette scores for clusters when merging is done, is not done, and for clusters produced by Splinter. The Silhouette score for a cluster is the average of all Silhouette scores of the items. The Silhouette score for an item is an intra-cluster similarity and inter-cluster dissimilarity score ranging from -1 to 1 , where -1 suggests that the item belongs to another cluster and 1 suggests that the item is only found in this cluster. For a more complete explanation, refer to Section 5.1.

For the Gamut_2 dataset, we failed to run Splinter's clustering to completion. This can be 


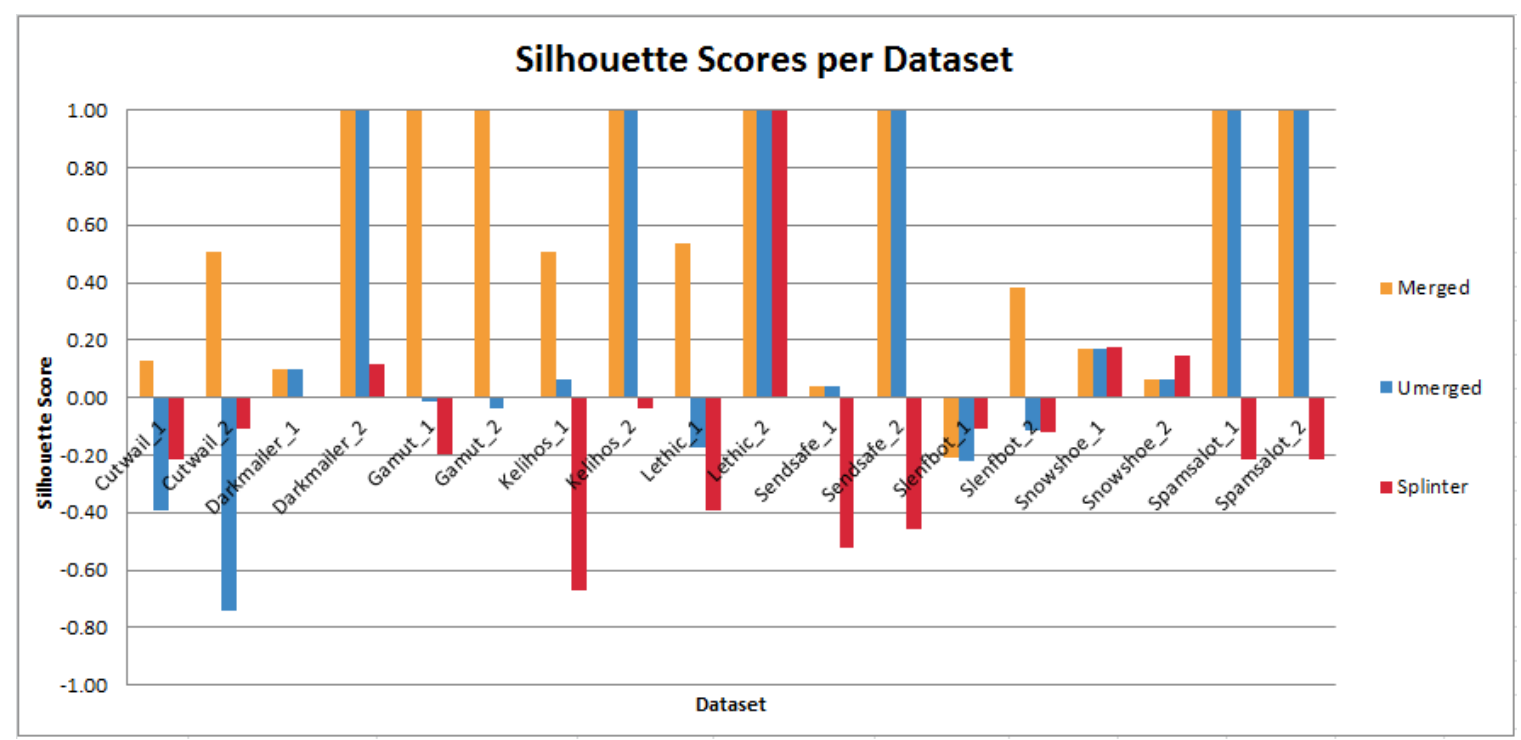

Figure 6.6: Silhouette scores for clusters produced by each system for each dataset.

attributed to the number of items this dataset produces. The number of clusters is set to -1 in these cases. The Silhouette score of 0 is assigned. Darkmailer_1 was parsed and clustered by Splinter, however the Silhouette score is assigned to 0 because we were unable to run to completion. Again, this can be attributed to the large number of items that this dataset produced. Aside from the aforementioned datasets, when merging was applied the Silhouette Scores did not decrease. In most cases the Silhouette scores increased for each of the datasets. This shows that it was merging similar clusters and producing more dissimilar clusters. Data used to create Figure 6.6 can be found in Appendix A.2 Table A.4.

The system determined 7 and 109 clusters with merging and without merging for Cutwail_1. Figure 6.7 emphasis that with merging, the Silhouette scores per cluster increase, indicating that the clusters are more dissimilar. The lower number of clusters can be explained by examining the graph containing 109 clusters. When checking the requirements, the system does not take into consideration the other already existing clusters, thus similar nodes on a neighboring branch are considered as part of another cluster. When merging is applied, the system takes into consideration the already existing clusters. When a new cluster is identified, it is checked for similiarity against 


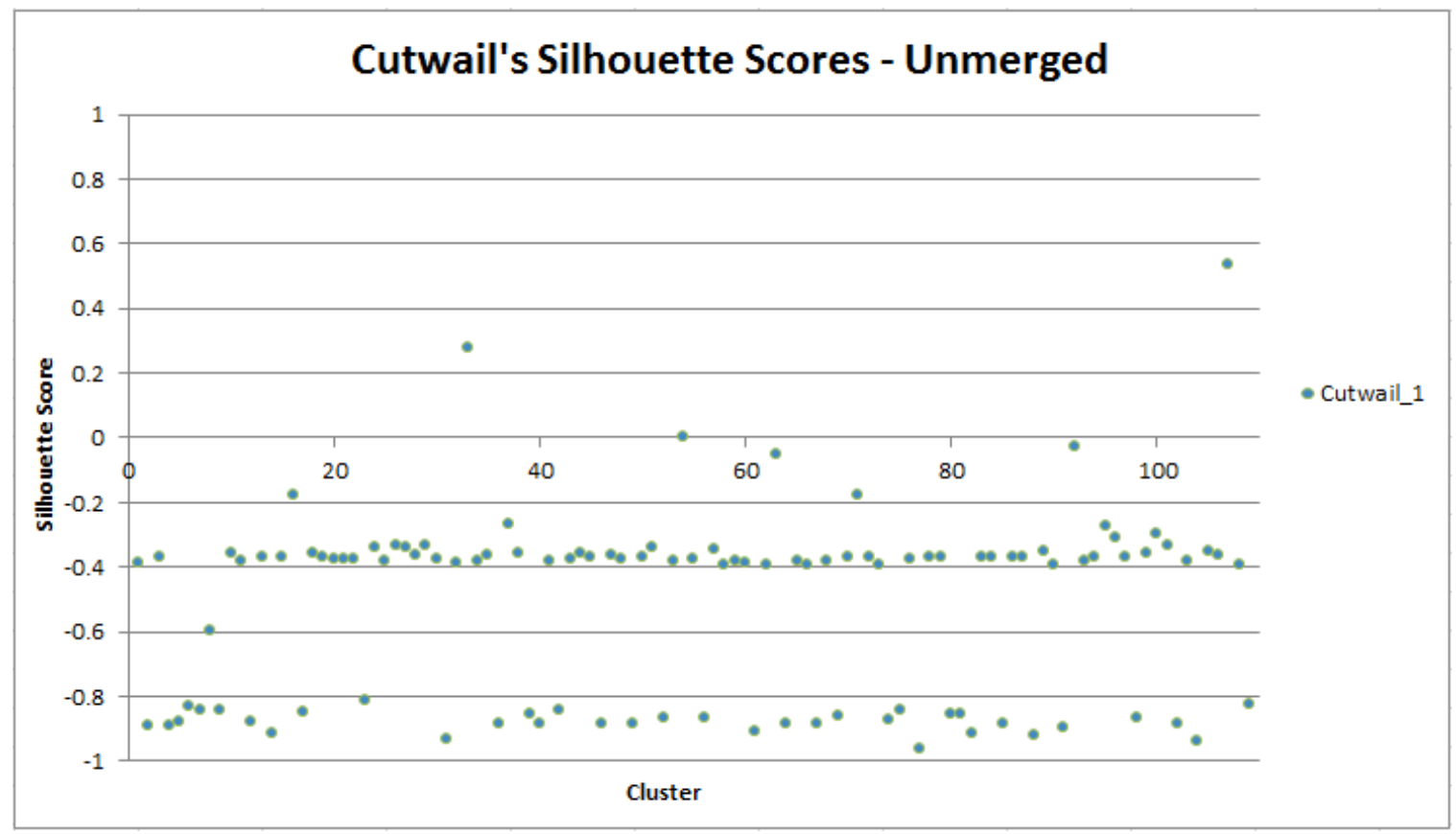

(a) Merging was not done.

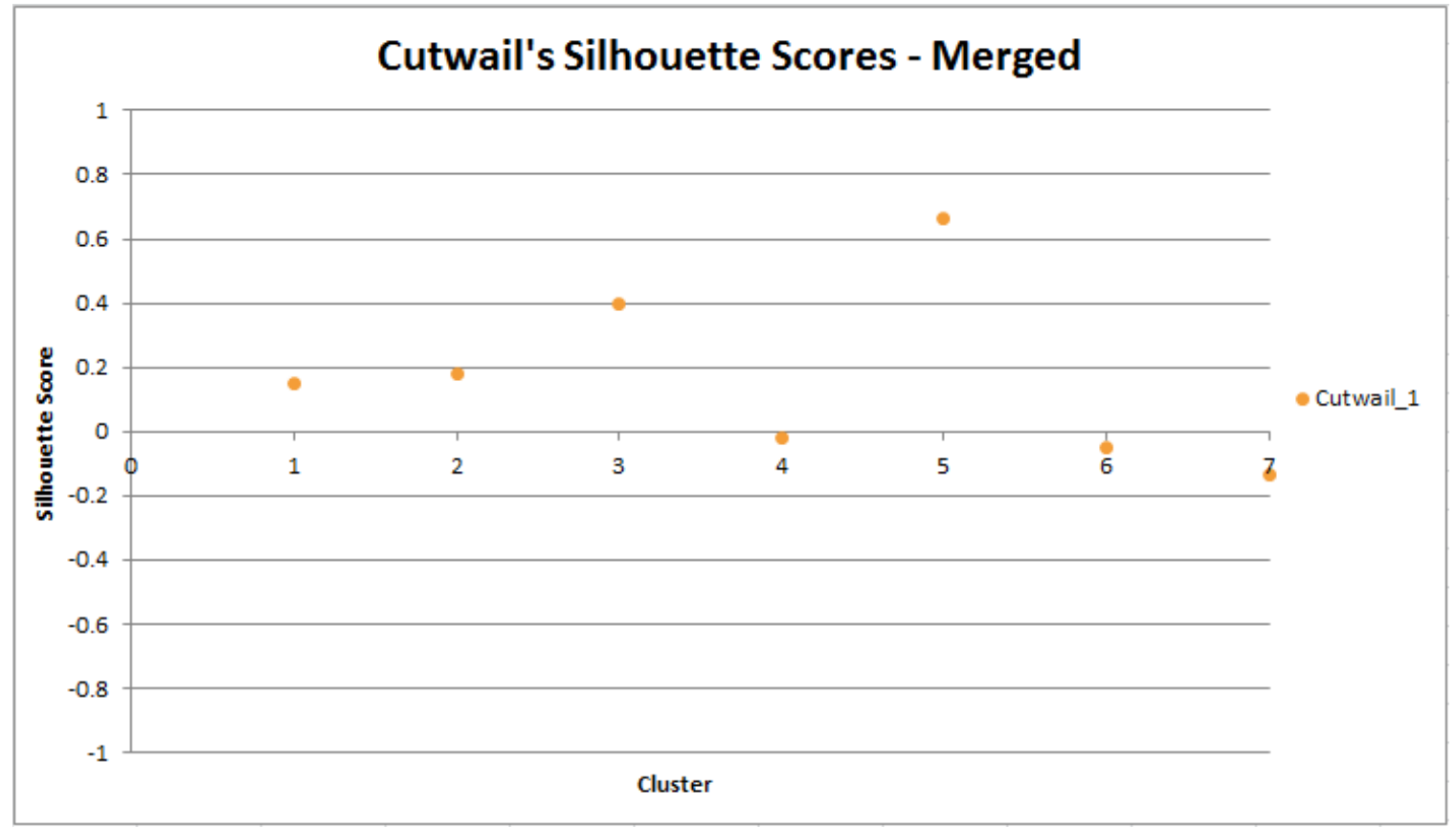

(b) Merging was done.

Figure 6.7: Cutwail's Silhouette scores for merged and unmerged clusters.

already existing clusters.

Table 6.2 presents additional datasets that were tested only with CAFE and not with Splinter. 
Table 6.2: Larger datasets.

\begin{tabular}{ccc} 
Date & Name & \# of Emails \\
\hline 20160120 & Lethic_3 & 3787300 \\
20160118 & Lethic_4 & 2555103 \\
20140627 & Unknown_2 & 2290860 \\
20150628 & Unknown_3 & 2285386 \\
20150627 & Unknown_4 & 2216174 \\
20141127 & Unknown_5 & 2142704 \\
20151202 & Slenfbot_3 & 1842996 \\
20160118 & Cutwail_3 & 520085 \\
20160120 & Cutwail_4 & 481742 \\
20151129 & Slenfbot_4 & 286976 \\
20160803 & Gamut_3 & 115660 \\
20151203 & Gamut_4 & 106700 \\
\hline
\end{tabular}

Majority of these datasets exceed the capability of Splinter due to resource restrictions of being a single process system and not distributed. When picking these datasets, the largest number of spam emails for each botnet was picked and subsequently the next largest. Four Unknown datasets were used, as this source provides consistently a large amount of spam emails. Figure 6.8 shows

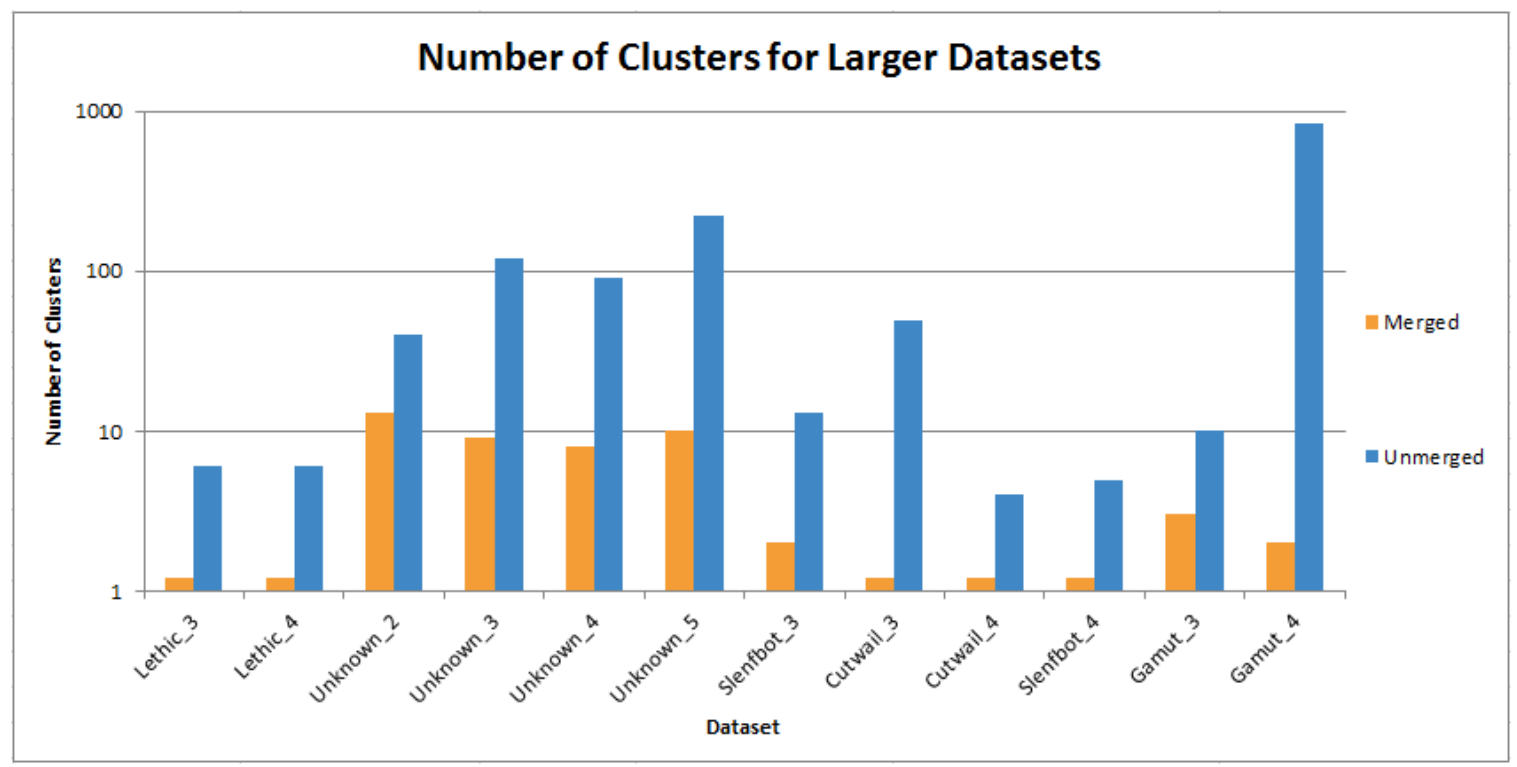

Figure 6.8: Number of clusters produced by each of the larger datasets by each variant of CAFE.

the number of clusters with and without cluster merging for each of the larger dataset. Detailed numbers can be found in Appendix A.3 Table A.1. This shows that CAFE is able to scale with an 
increase of spam emails, and further reinforces that it is capable of merging similar clusters.

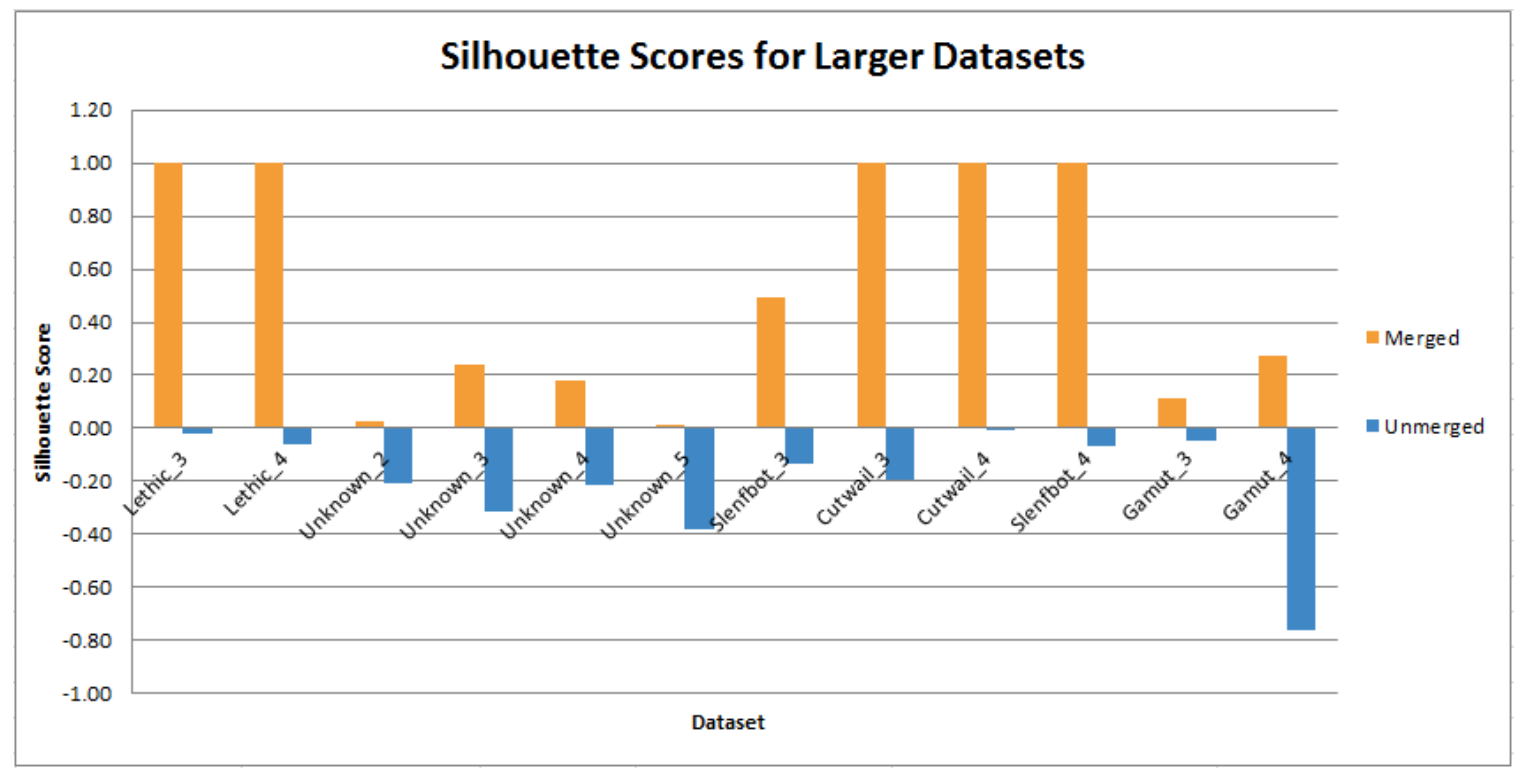

Figure 6.9: Silhouette scores for clusters produced by each of the larger datasets by each variant of CAFE.

Each dataset was scored using the Silhouette score for both instances, with and without merging. Figure 6.9 shows the Silhouette scores, further reinforcing that when merging was applied to the larger datasets it consistantly improves Silhouette scores. The exact numerical values can be found in Appendix A.3 Table A.2.

Based on the clusters produced by the larger datasets, the following observations were made. Both Lethic datasets have one cluster when merging is applied. As previously mentioned in Section 6.1 Lethic only has one type of spam, which is an HTML message about selling luxury items such as watches and bags. Both Cutwail datasets identified one cluster when merging was applied, while truly containing three types. Visual inspection of the dataset reveals that there was in fact one type of spam emails that is very closely resembling that of Lethic.

For one Slenfbot dataset, CAFE identifies one cluster. This corresponding to the type mentioned in Section 6.1. The other Slenfbot dataset identifies two clusters. This possibly means that for that dataset there is an additional type of Slenfbot. Visual inspection of the dataset for which two 
clusters were identified shows that Slenfbot was in fact sending a second type of spam emails. The second type of spam is a plain text message about available job positions that also provides an email address to reply to.

Gamut identifies two and three clusters, while truly having four types. It is worth mentioning that while the prevalent type of spam for a botnet may relatively be the same throughout the lifespan of the botnet, the other less prevalent types may happen seemingly sporadically. CAFE produced three clusters for Gamut_3. Visual inspection reveals that they are all of one type, which was the fourth type about job offers. The reason for being identified as distinct clusters is aside from being about job offers and maintaining the multi-part message composed of plain text and HTML, the body layouts and subjects change significantly between the three clusters. Visual inspection showed that the dataset for Gamut_4 only contained two types. The first type is about hooking up with an encoded message body. The second type is about also hooking up but without an encoded message body. The other two of the four types were not present.

In conclusion, merging is recommended in order to produce more dissimilar clusters. Without merging being done more clusters are identified. However, their inspection reveals that they are mutually similar.

\subsection{Performance}

Performance is compared using the same datasets as in Section 6.2. Time was recorded in seconds and is the summation of both parsing and clustering. First, processing time of CAFE is compared to Splinter's processing time. Followed by showing scaling by processing larger datasets that Splinter is unable to handle.

Figure 6.10a shows the total processing time in seconds by CAFE. Figure 6.10b shows the total processing time in seconds for Splinter. Only both of the Spamsalot datasets took longer than 400 seconds when processed by CAFE and when merging had been applied. This can be attributed to 


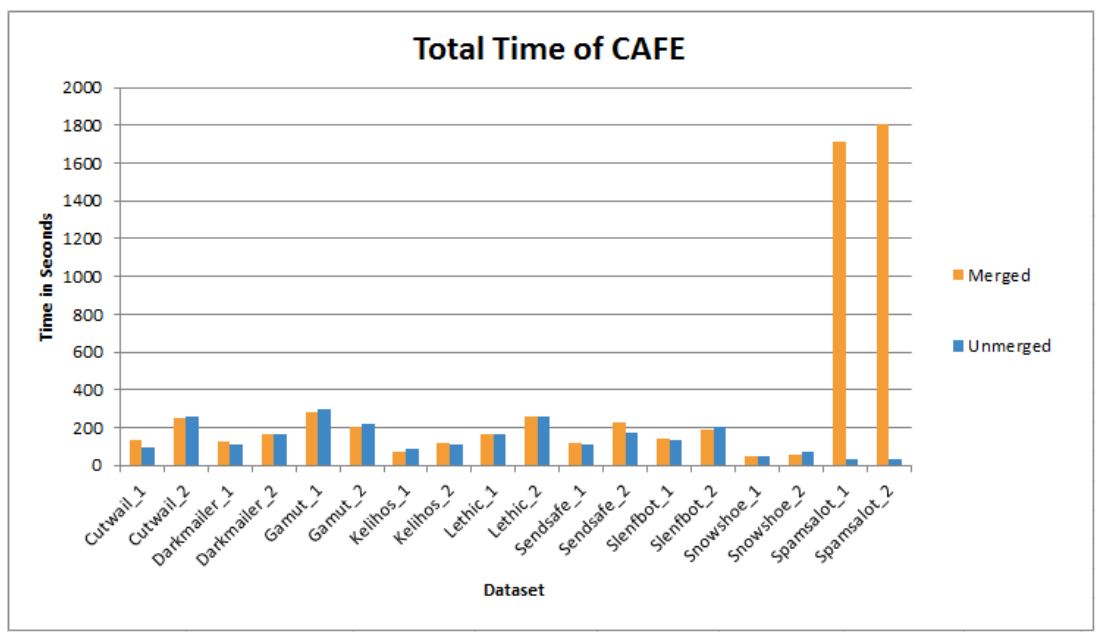

(a) CAFE's parsing and clustering total time for both variants.

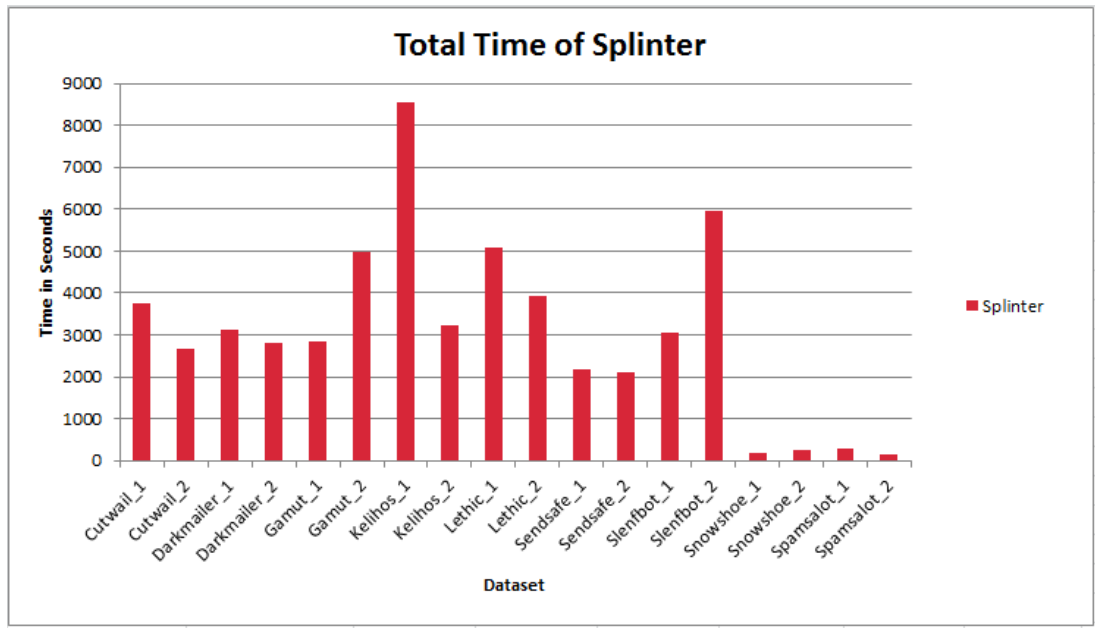

(b) Splinter's parsing and clustering total time.

Figure 6.10: Total time for Splinter and CAFE.

the number of items produced by those dataset as well as the time cost of using Silhouette scoring for merging. With the exception of both of the Snowshoe and Spamsalot datasets Splinter, took well over 1000 seconds. Exact processing times and a break down between parsing and clustering can be found in Appendix A.3 Table A.5, Table A.7, and Table A.6. It is worth mentioning that the vast difference between Splinter's processing time and the system presented in this thesis lays mainly in Splinter's parsing and not the clustering. While Splinter's clustering time ranges between 3 and 227 seconds, the parsing time ranges between 108 and 4896 seconds, averaging at 2871 seconds. This can be attributed to Splinter being a single threaded program with insertions to a local database on 
per email basis. This also prevents it from scaling beyond the limits of the machine that is being ran on.

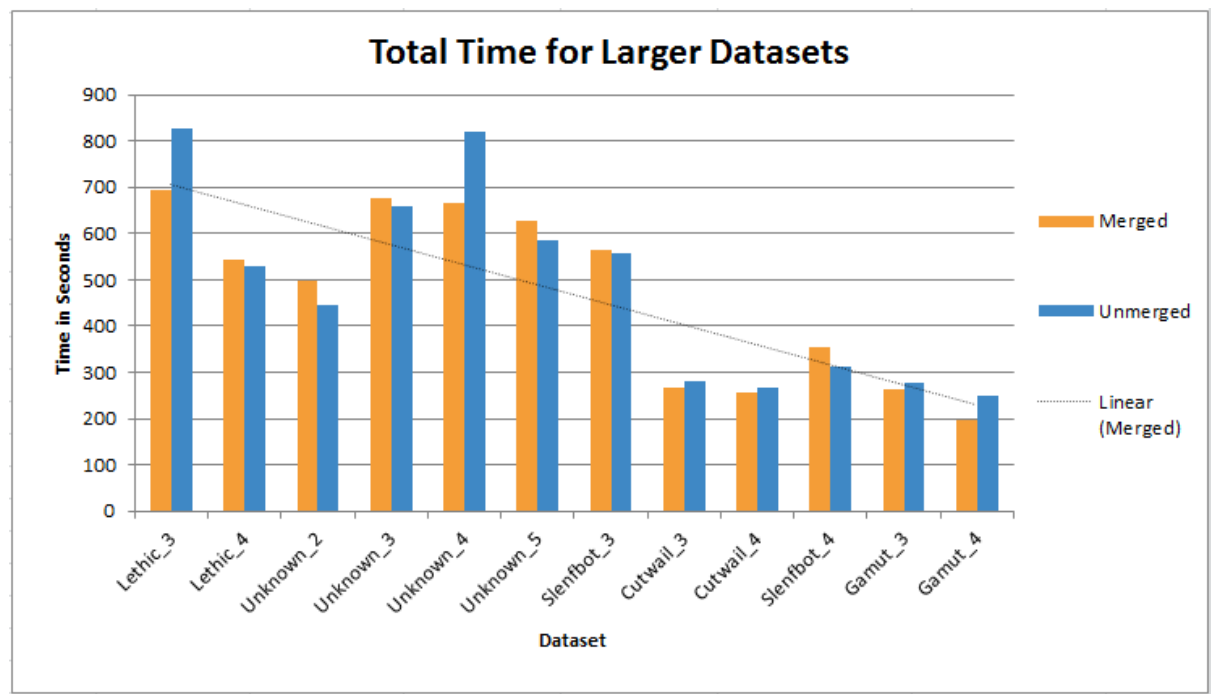

Figure 6.11: Total time for parsing and clustering each of the larger dataset by each variant of CAFE

Figure 6.11 shows the total processing time for larger datasets. While the first seven datasets range between 1.8 to 3 million emails, the last five datasets range between 106 to 520 thousand emails. The sudden drop between the two groups is expected, while also showing that within certain amounts of emails the processing time varies little.

In conclusion, the use of Hadoop to parallelize and distribute the parsing of spam emails, creation of the tree, as well as clustering enables the system to handle large datasets, thus scaling, and processing time faster than on a single machine, single thread program. 


\section{Chapter 7}

\section{Conclusion}

Spam emails are a current concern. Due to their sheer volume, it is a tedious task for investigators to analyze. Systems and tools are necessary to overcome the tediousness and assist the investigators in their work. In this thesis, we presented a system, named CAFE, that can process and cluster spam emails using FP-Trees and the distributed computing framework Hadoop. Clustering spam emails using FP-Tree was inspired by Splinter, a system presented by Dihn et al. [2]. Splinter is not able to scale well due to being a single process running on a single machine. It also produces clusters that are similar to each other. To overcome these two issues, the algorithm used by Splinter that utilizes the FP-Tree data structure had been improved in order to produce more dissimilar clusters while also leveraging Hadoop.

In Chapter 4, we explained the system design in detail, showing how CAFE is constructed to work on Hadoop. Specifically, in Section 4.5, we talked about optimizations made to CAFE to both increase the performance and to cluster better. The main optimization was is clusters. It reduces the number of clusters while making them more dissimilar. Chapter 6 presents the experiments ran and their results. Figures 6.5 and 6.6 illustrate this improvement, showing that when merging is done, less and more dissimlar clusters are detected. Figure 6.10 shows the total processing times of Splinter and CAFE. CAFE processed most datasets under 400 seconds while Splinter took longer 
than 1000 seconds on most datasets, showing the performance improvement of utilizing Hadoop. Figure 6.11 shows the scaling that has been gained through utilizing Hadoop. Larger datasets can be processed and the time scales based on size.

At the time of this writing, CAFE does not process live data. Instead, it processes data collected from the previous day. Thus, it is constantly one day behind. One improvement could be to make the spam ingestion handle live streaming data. This change would greatly impact the system design from that point onwards. Each piece of the system will be required to change to accommodate. This can be done by using NiFi or Spark, which run on top of Hadoop. Spark allows for stream processing while NiFi can provide the connections for the streams. The stream in this case would be a direct feed of spam emails as they arrive.

While clusters are identified, they are not classified based on subjective categories. Creating an extra process that would classify the identified clusters is something that could be done.

Additional cluster algorithms could be used to do one of the following two things. The results from additional clustering algorithms could further verify how well the base algorithm works. Several algorithms could also be used to overlap one another. The intersection of all the algorithms used would then be a more concrete result. For instance, let algorithm A identifies cluster $C_{1}$ consisting of a set of emails $\mathrm{X}$ while algorithm $\mathrm{B}$ identifies $C_{2}$ consisting of a set of emails $\mathrm{Y}$. The intersection of $\mathrm{X}$ and $\mathrm{Y}$ produces a set of emails that both algorithm $\mathrm{A}$ and $\mathrm{B}$ recognized as a cluster, thus reaffirming that those emails are similar. Possible candidates would be the algorithm presented by Shoeb et al. [7] and K-Means [15] clustering algorithm. The algorithm presented by Shoeb et al. [7] takes a unique approach, based on the redirection of a URL. K-means could also be used using Apache Mahout, which runs on Hadoop. K-Mean would cluster based on the whole email. The down side to K-means is the need to specify the number of centroids. The number of centroids could be set to the number of types per botnet mentioned in Section 6.1. However, when the dataset is not attributed to a botnet, contains a new type, or does not contain some of the known types, it is harder to pick the number of centroids, thus yeilding less than optimal results. 
Branches in the FP-Tree represent itemsets. These itemsets are sorted based on the number of times an item occurrs in the dataset. Items that occur more often appear closer to the root. Those occurring less often are further down in the tree. Assignment of weights to items and sorting itemsets based on weights as opposed to the number of occurrences can be explored in future works. This weight could be a linear function of the number of occurrences times a multiplier specific to the item or its type. This would potentially bring more interesting items such as hostnames or MD5s of attachments closer to the root of the tree. The multiplier would greatly benefit from input of expert knowledge. 


\section{Appendix A}

\section{Collected Metrics}

\section{A.1 Clusters and Silhouette Scores for Larger Datasets}

These tables present more detailed data for datasets that are used only by CAFE. The number of clusters detected for larger datasets can be found in Table A.1. The Silhouette coefficients for the larger datasets can be found in Table A.2.

Table A.1: Larger datasets metrics.

\begin{tabular}{cccccc} 
Name & $\begin{array}{c}\text { Minimum } \\
\text { Support }\end{array}$ & Nodes & $\begin{array}{c}\text { Emails } \\
\text { on graph }\end{array}$ & $\begin{array}{c}\text { Clusters } \\
\text { with Merging }\end{array}$ & $\begin{array}{c}\text { Clusters } \\
\text { without Merging }\end{array}$ \\
\hline Lethic_3 & 4261 & 104464 & 3787300 & 1 & 6 \\
Lethic_4 & 2874 & 113598 & 2555102 & 1 & 6 \\
Unknown_2 & 2577 & 4316 & 2290190 & 13 & 40 \\
Unknown_3 & 2571 & 4765 & 2285382 & 9 & 121 \\
Unknown_4 & 2493 & 4465 & 2216169 & 8 & 91 \\
Unknown_5 & 2411 & 32242 & 2142665 & 10 & 223 \\
Slenfbot_3 & 2073 & 732 & 1842920 & 2 & 13 \\
Cutwail_3 & 585 & 249843 & 520073 & 1 & 49 \\
Cutwail_4 & 542 & 180159 & 481684 & 1 & 4 \\
Slenfbot_4 & 323 & 82 & 286857 & 1 & 5 \\
Gamut_3 & 130 & 6963 & 115660 & 3 & 10 \\
Gamut_4 & 120 & 24965 & 106698 & 2 & 829 \\
\hline
\end{tabular}


Table A.2: Silhouette scores for larger datasets.

\begin{tabular}{cccc} 
Name & Merged & Unmerged & $\begin{array}{c}\text { Score } \\
\text { Difference }\end{array}$ \\
\hline Lethic_3 & 1.00 & -0.02 & 1.02 \\
Lethic_4 & 1.00 & -0.06 & 1.06 \\
Unknown_2 & 0.02 & -0.21 & 0.23 \\
Unknown_3 & 0.24 & -0.31 & 0.55 \\
Unknown_4 & 0.18 & -0.21 & 0.40 \\
Unknown_5 & 0.01 & -0.38 & 0.39 \\
Slenfbot_3 & 0.50 & -0.13 & 0.63 \\
Cutwail_3 & 1.00 & -0.19 & 1.19 \\
Cutwail_4 & 1.00 & -0.01 & 1.01 \\
Slenfbot_4 & 1.00 & -0.07 & 1.07 \\
Gamut_3 & 0.11 & -0.05 & 0.16 \\
Gamut_4 & 0.27 & -0.76 & 1.04 \\
\hline
\end{tabular}

\section{A.2 Clusters and Silhouette Scores for Both Systems}

The following tables present more detailed data for datasets that are used by both CAFE and Splinter. Number of clusters detected by each dataset for each variation can be found in Table A.3. The Silhouette coefficients for clusters produced by both systems can be found in Table A.4.

Table A.3: Cluster and graph metrics for Splinter and both variants of CAFE.

\begin{tabular}{ccccccc} 
Name & $\begin{array}{c}\text { Minimum } \\
\text { Support }\end{array}$ & Nodes & $\begin{array}{c}\text { Emails } \\
\text { on Graph }\end{array}$ & $\begin{array}{c}\text { Clusters } \\
\text { with Merging }\end{array}$ & $\begin{array}{c}\text { Clusters } \\
\text { without Merging }\end{array}$ & $\begin{array}{c}\text { Clusters } \\
\text { by Splinter }\end{array}$ \\
\hline Cutwail_1 & 117 & 11515 & 103565 & 11 & 111 & 682 \\
Cutwail_2 & 113 & 28779 & 101296 & 1 & 313 & 554 \\
Darkmailer_1 & 75 & 146 & 67400 & 2 & 2 & 4 \\
Darkmailer_2 & 69 & 128 & 61078 & 1 & 1 & 3 \\
Gamut_1 & 121 & 4216 & 107025 & 1 & 40 & 663 \\
Gamut_2 & 121 & 55 & 107730 & 1 & 6 & -1 \\
Kelihos_1 & 113 & 1132 & 100878 & 3 & 5 & 2775 \\
Kelihos_2 & 83 & 161 & 74199 & 1 & 1 & 6 \\
Lethic_1 & 121 & 2053 & 107470 & 2 & 16 & 34 \\
Lethic_2 & 111 & 32653 & 99274 & 1 & 1 & 1 \\
Sendsafe_1 & 0 & 556 & 32507 & 13 & 13 & 77 \\
Sendsafe_2 & 56 & 44 & 49660 & 1 & 1 & 5 \\
Slenfbot_1 & 70 & 205 & 62529 & 15 & 16 & 3 \\
Slenfbot_2 & 55 & 1471 & 48460 & 2 & 20 & 4 \\
Snowshoe_1 & 0 & 8990 & 3325 & 6 & 6 & 3 \\
Snowshoe_2 & 0 & 8863 & 3278 & 4 & 4 & 3 \\
Spamsalot_1 & 0 & 12 & 1895 & 1 & 1 & 3 \\
Spamsalot_2 & 0 & 12 & 2403 & 1 & 1 & \\
\hline
\end{tabular}


Table A.4: Silhouette scores for Splinter and both variants of CAFE.

\begin{tabular}{cccccc} 
Name & Merged & Umerged & $\begin{array}{c}\text { Score } \\
\text { Difference }\end{array}$ & Splinter & $\begin{array}{c}\text { Splinter } \\
\text { vs } \\
\text { Merged }\end{array}$ \\
\hline Cutwail_1 & 0.13 & -0.39 & 0.52 & -0.22 & 0.34 \\
Cutwail_2 & 0.51 & -0.74 & 1.25 & -0.11 & 0.61 \\
Darkmailer_1 & 0.10 & 0.10 & 0.00 & 0.00 & 0.10 \\
Darkmailer_2 & 1.00 & 1.00 & 0.00 & 0.11 & 0.89 \\
Gamut_1 & 1.00 & -0.01 & 1.01 & -0.20 & 1.20 \\
Gamut_2 & 1.00 & -0.04 & 1.04 & 0.00 & 1.00 \\
Kelihos_1 & 0.50 & 0.06 & 0.44 & -0.67 & 1.17 \\
Kelihos_2 & 1.00 & 1.00 & 0.00 & -0.03 & 1.03 \\
Lethic_1 & 0.53 & -0.17 & 0.71 & -0.39 & 0.92 \\
Lethic_2 & 1.00 & 1.00 & 0.00 & 1.00 & 0.00 \\
Sendsafe_1 & 0.04 & 0.04 & 0.00 & -0.52 & 0.56 \\
Sendsafe_2 & 1.00 & 1.00 & 0.00 & -0.45 & 1.45 \\
Slenfbot_1 & -0.21 & -0.22 & 0.01 & -0.11 & -0.10 \\
Slenfbot_2 & 0.39 & -0.11 & 0.50 & -0.12 & 0.51 \\
Snowshoe_1 & 0.17 & 0.17 & 0.00 & 0.18 & -0.01 \\
Snowshoe_2 & 0.06 & 0.06 & 0.00 & 0.14 & -0.08 \\
Spamsalot_1 & 1.00 & 1.00 & 0.00 & -0.21 & 1.21 \\
Spamsalot_2 & 1.00 & 1.00 & 0.00 & -0.21 & 1.21 \\
\hline
\end{tabular}

\section{A.3 Performance Metrics}

The tables found here give more detailed data on performances by both CAFE and Splinter. All recorded times are in seconds. The total processing times by both CAFE and Splinter can be found in Table A.5. The breakdown of processing time for CAFE can be found in Table A.6. Splinter's processing time breakdown can be found in Table A.7. The breakdown of processing time by CAFE for the larger datasets can be found in Table A.8. 
Table A.5: Total processing times in seconds.

\begin{tabular}{cccc} 
Name & Merged & Unmerged & Splinter \\
\hline Cutwail_1 & 137 & 98 & 3742 \\
Cutwail_2 & 247 & 261 & 2666 \\
Darkmailer_1 & 124 & 109 & 3113 \\
Darkmailer_2 & 167 & 165 & 2822 \\
Gamut_1 & 280 & 301 & 2836 \\
Gamut_2 & 201 & 221 & 4975 \\
Kelihos_1 & 73 & 85 & 8562 \\
Kelihos_2 & 121 & 109 & 3427 \\
Lethic_1 & 167 & 165 & 5069 \\
Lethic_2 & 259 & 259 & 4584 \\
Sendsafe_1 & 117 & 110 & 2176 \\
Sendsafe_2 & 229 & 170 & 2113 \\
Slenfbot_1 & 144 & 134 & 2888 \\
Slenfbot_2 & 191 & 203 & 2238 \\
Snowshoe_1 & 51 & 51 & 167 \\
Snowshoe_2 & 55 & 72 & 237 \\
Spamsalot_1 & 1713 & 35 & 271 \\
Spamsalot_2 & 1804 & 35 & 111 \\
\hline
\end{tabular}

Table A.6: CAFE's processing times.

\begin{tabular}{cccccc} 
Name & Parsing Time & $\begin{array}{c}\text { Clustering Time } \\
\text { with } \\
\text { Merging }\end{array}$ & $\begin{array}{c}\text { Clustering Time } \\
\text { without } \\
\text { Merging }\end{array}$ & $\begin{array}{c}\text { Total Time } \\
\text { with } \\
\text { Merging }\end{array}$ & $\begin{array}{c}\text { Total Time } \\
\text { without } \\
\text { Merging }\end{array}$ \\
\hline Cutwail_1 & 69 & 68 & 29 & 137 & 98 \\
Cutwail_2 & 196 & 51 & 65 & 247 & 261 \\
Darkmailer_1 & 79 & 45 & 30 & 124 & 109 \\
Darkmailer_2 & 133 & 35 & 33 & 167 & 165 \\
Gamut_1 & 260 & 20 & 41 & 280 & 301 \\
Gamut_2 & 178 & 23 & 43 & 201 & 221 \\
Kelihos_1 & 47 & 26 & 39 & 73 & 85 \\
Kelihos_2 & 90 & 31 & 19 & 121 & 109 \\
Lethic_1 & 142 & 24 & 23 & 167 & 165 \\
Lethic_2 & 235 & 25 & 24 & 259 & 259 \\
Sendsafe_1 & 94 & 24 & 17 & 117 & 110 \\
Sendsafe_2 & 150 & 78 & 19 & 229 & 170 \\
Slenfbot_1 & 118 & 26 & 16 & 144 & 134 \\
Slenfbot_2 & 147 & 44 & 56 & 191 & 203 \\
Snowshoe_1 & 23 & 28 & 28 & 51 & 51 \\
Snowshoe_2 & 23 & 33 & 49 & 55 & 72 \\
Spamsalot_1 & 19 & 1694 & 15 & 1713 & 35 \\
Spamsalot_2 & 21 & 1782 & 14 & 1804 & 35 \\
\hline
\end{tabular}


Table A.7: Splinter's processing times in seconds.

\begin{tabular}{cccc} 
Name & $\begin{array}{c}\text { Splinter's } \\
\text { Parsing Time }\end{array}$ & $\begin{array}{c}\text { Splinter's } \\
\text { Clustering Time }\end{array}$ & $\begin{array}{c}\text { Splinter's } \\
\text { Total Time }\end{array}$ \\
\hline Cutwail_1 & 3654 & 88 & 3742 \\
Cutwail_2 & 2542 & 124 & 2666 \\
Darkmailer_1 & 2736 & 86 & 3113 \\
Darkmailer_2 & 2736 & 86 & 2822 \\
Gamut_1 & 2750 & 86 & 2836 \\
Gamut_2 & 4824 & 151 & 4975 \\
Kelihos_1 & 8335 & 227 & 8562 \\
Kelihos_2 & 3323 & 104 & 3427 \\
Lethic_1 & 4896 & 173 & 5069 \\
Lethic_2 & 4445 & 139 & 4584 \\
Sendsafe_1 & 2137 & 39 & 2176 \\
Sendsafe_2 & 2007 & 106 & 2113 \\
Slenfbot_1 & 2800 & 88 & 2888 \\
Slenfbot_2 & 2170 & 68 & 2238 \\
Snowshoe_1 & 161 & 6 & 167 \\
Snowshoe_2 & 227 & 10 & 237 \\
Spamsalot_1 & 268 & 3 & 271 \\
Spamsalot_2 & 108 & 3 & 111 \\
\hline
\end{tabular}

Table A.8: Processing times for larger datasets.

\begin{tabular}{cccccc} 
Name & Parsing Time & $\begin{array}{c}\text { Clustering } \\
\text { with } \\
\text { Merging }\end{array}$ & $\begin{array}{c}\text { Clustering } \\
\text { without } \\
\text { Merging }\end{array}$ & $\begin{array}{c}\text { Total } \\
\text { with } \\
\text { Merging }\end{array}$ & $\begin{array}{c}\text { Total } \\
\text { without } \\
\text { Merging }\end{array}$ \\
\hline Lethic_3 & 605 & 89 & 220 & 694 & 826 \\
Lethic_4 & 432 & 110 & 97 & 542 & 529 \\
Unknown_2 & 374 & 125 & 73 & 499 & 447 \\
Unknown_3 & 575 & 100 & 85 & 675 & 660 \\
Unknown_4 & 596 & 70 & 223 & 666 & 819 \\
Unknown_5 & 509 & 120 & 76 & 628 & 584 \\
Slenfbot_3 & 500 & 65 & 57 & 565 & 556 \\
Cutwail_3 & 212 & 56 & 70 & 268 & 282 \\
Cutwail_4 & 197 & 58 & 69 & 255 & 267 \\
Slenfbot_4 & 292 & 61 & 19 & 353 & 311 \\
Gamut_3 & 216 & 48 & 60 & 264 & 276 \\
Gamut_4 & 171 & 27 & 80 & 198 & 251 \\
\hline
\end{tabular}




\section{A.4 Parser MapReduce Pseudocode}

The following are procedures in pseudocode for the Parser MapReduce job. The Map method is shown in Procedure ParserMap. The auxiliary Combiner method is provided in Prodecure ParserCombine. Procedure ParserPartitioner shows the auxiliary Partitioner method. The pseudocode for the Reduce method is provided in Procedure ParserReduce.
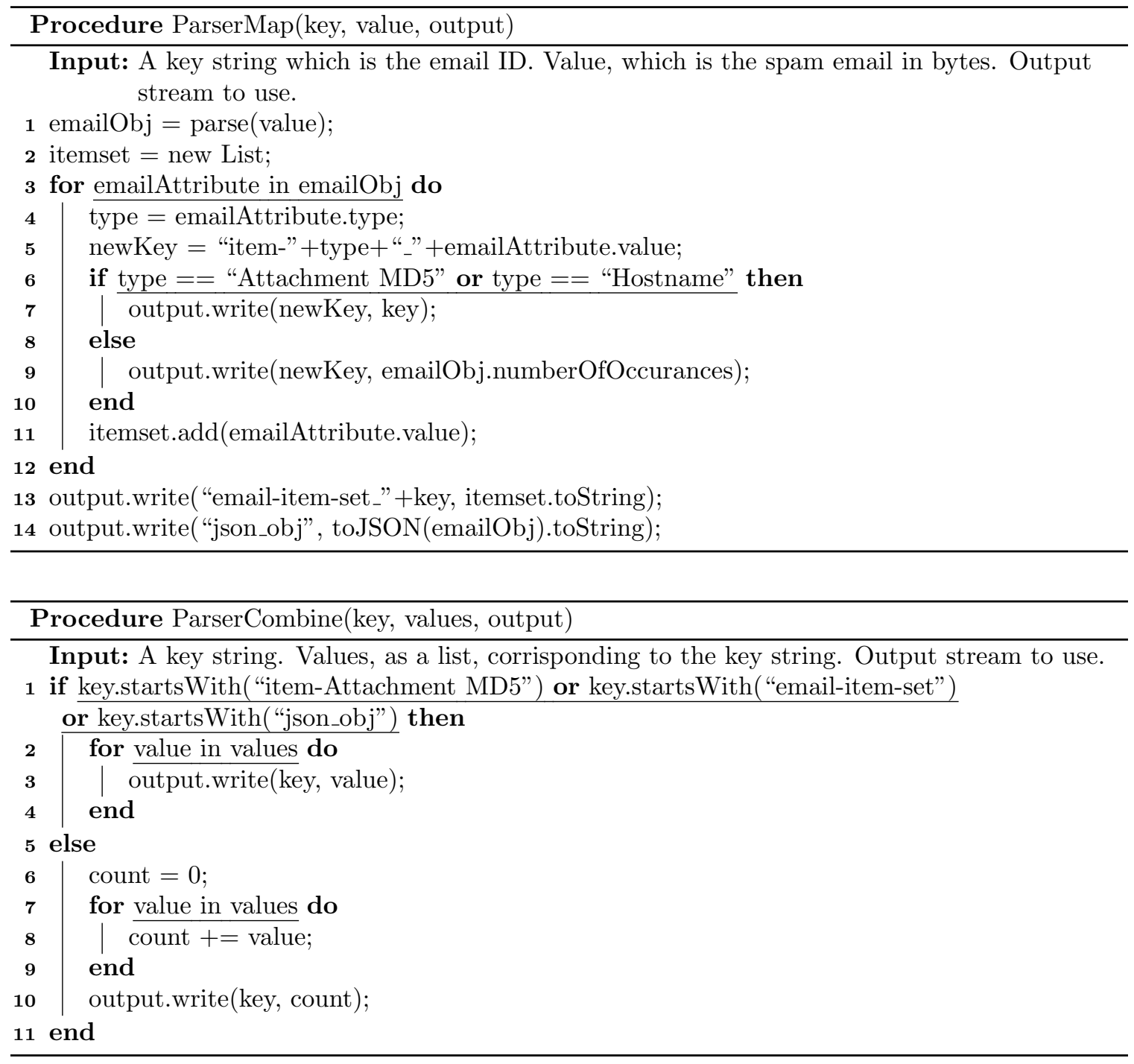

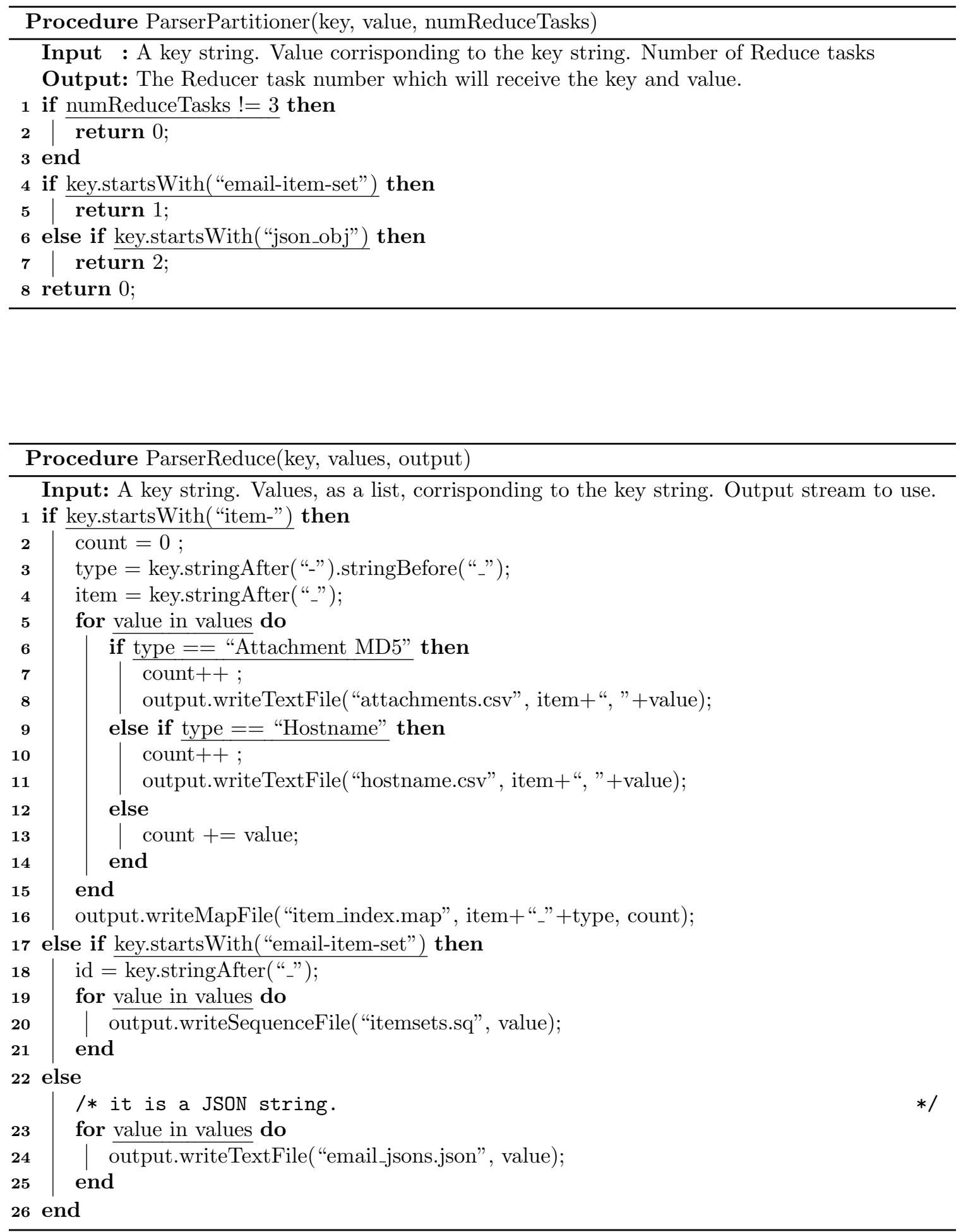


\section{A.5 TreeMaker MapReduce Pseudocode}

The following are procedures in pseudocode for both of TreeMaker's MapReduce jobs. The Map method for the first MapReduce job is shown in Procedure MegaSetMap. The auxiliary Combiner method for the first MapReduce job is provided in Procedure MegaSetCombiner. Pseudocode for the Reduce method of the first MapReduce job is shown in Procedure MegaSetReduce. The Map method for the second MapReduce job is shown in Procedure TreeMap. The pseudocode for the Reduce method of the second MapReduce job is provided in Procedure TreeReduce, the clean up method accompanying the Reduce method is shown in Procedure TreeReduceCleanUp. The method that identifies clusters if found in Procedure IdentifyCluster.
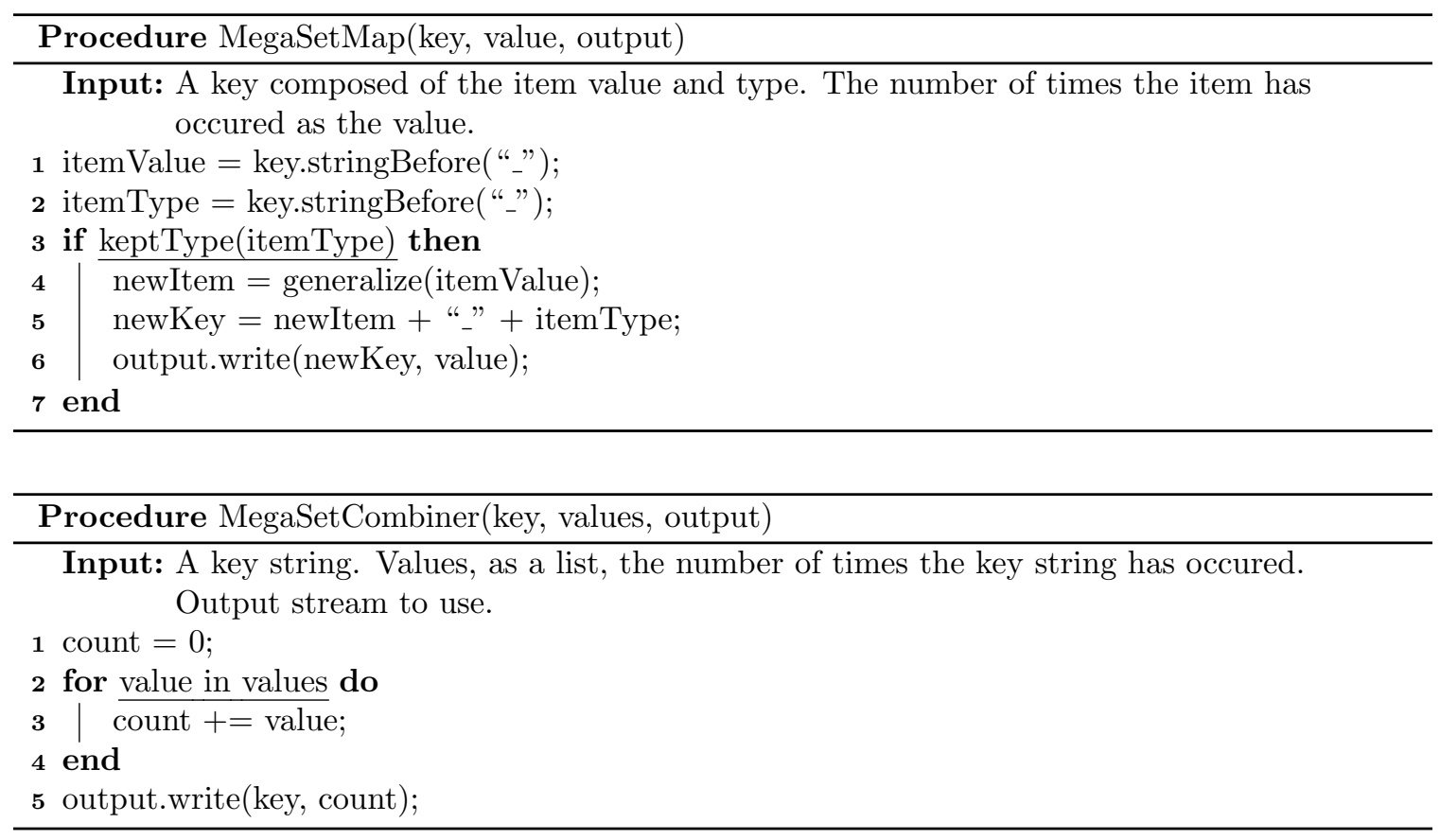


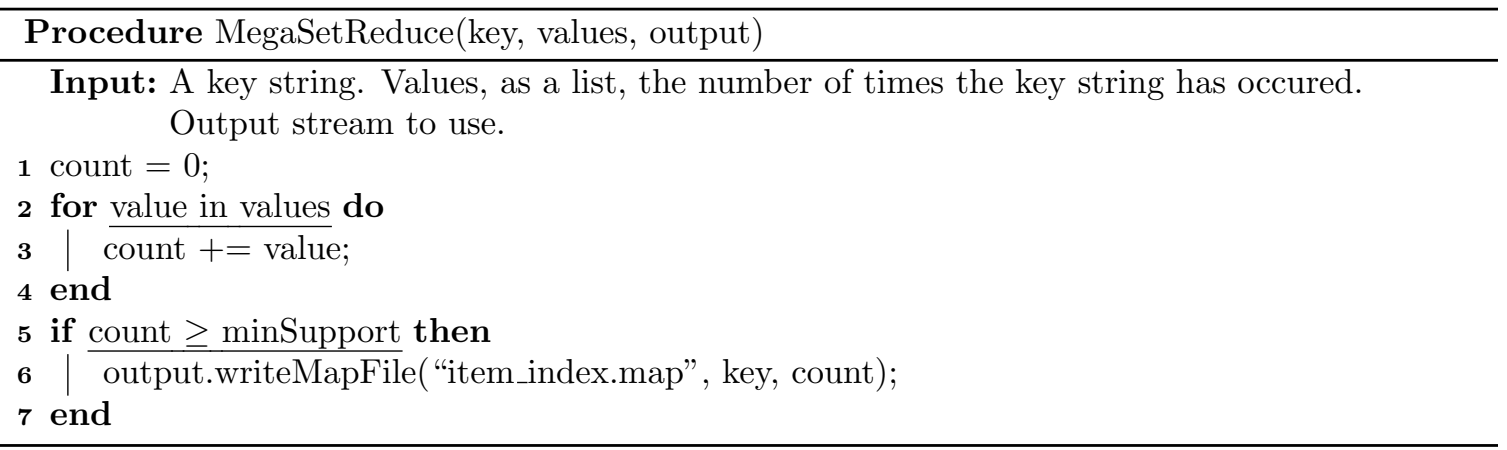

Procedure TreeMap(key, value, output)

Input: A key string which is the email ID. Value, which is the itemset. Output stream to use.

1 itemset $=$ parseItemset(value);

2 itemset $=$ sortitemset(itemset);

3 output.write(itemset.toString, key);

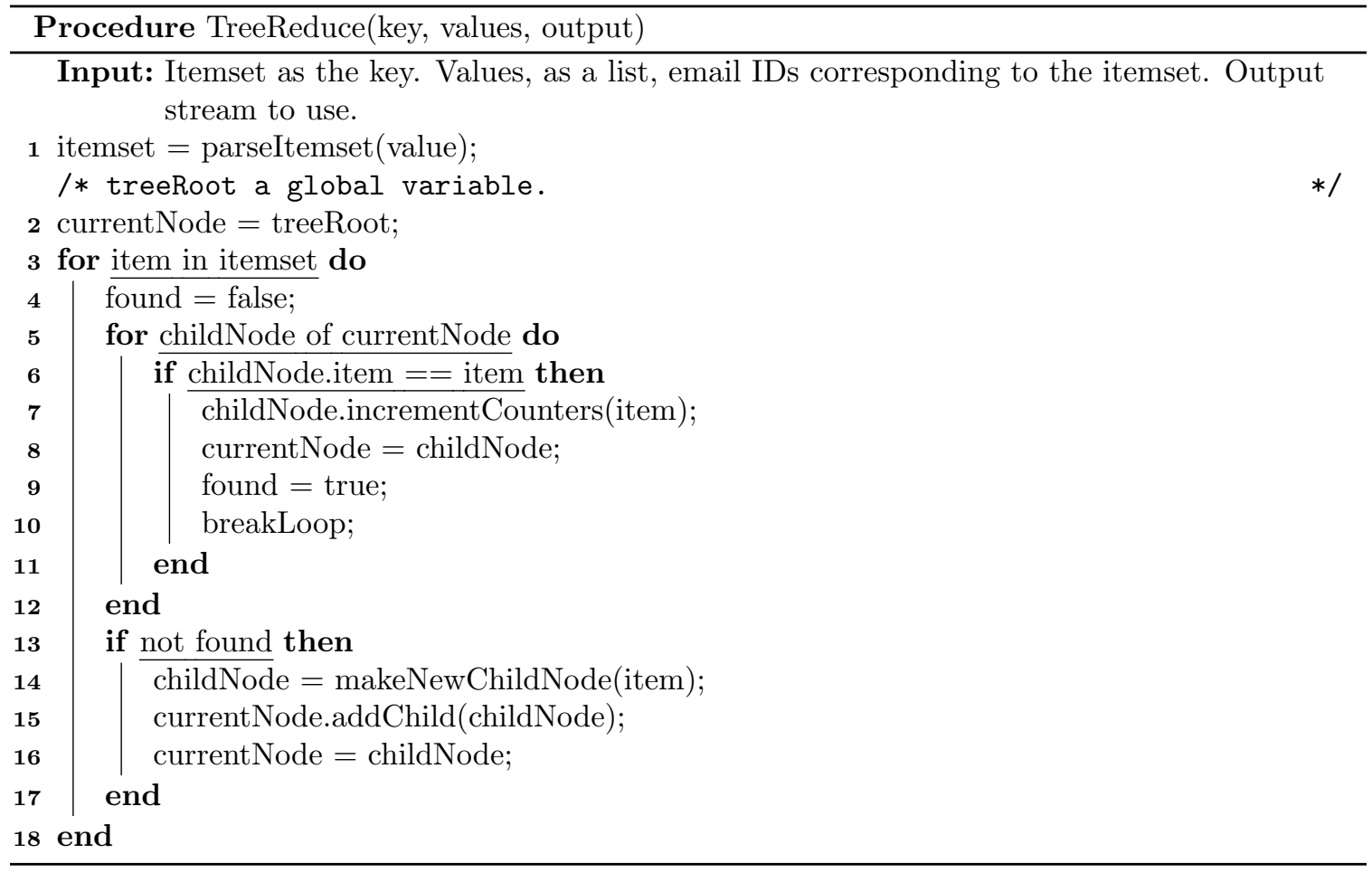



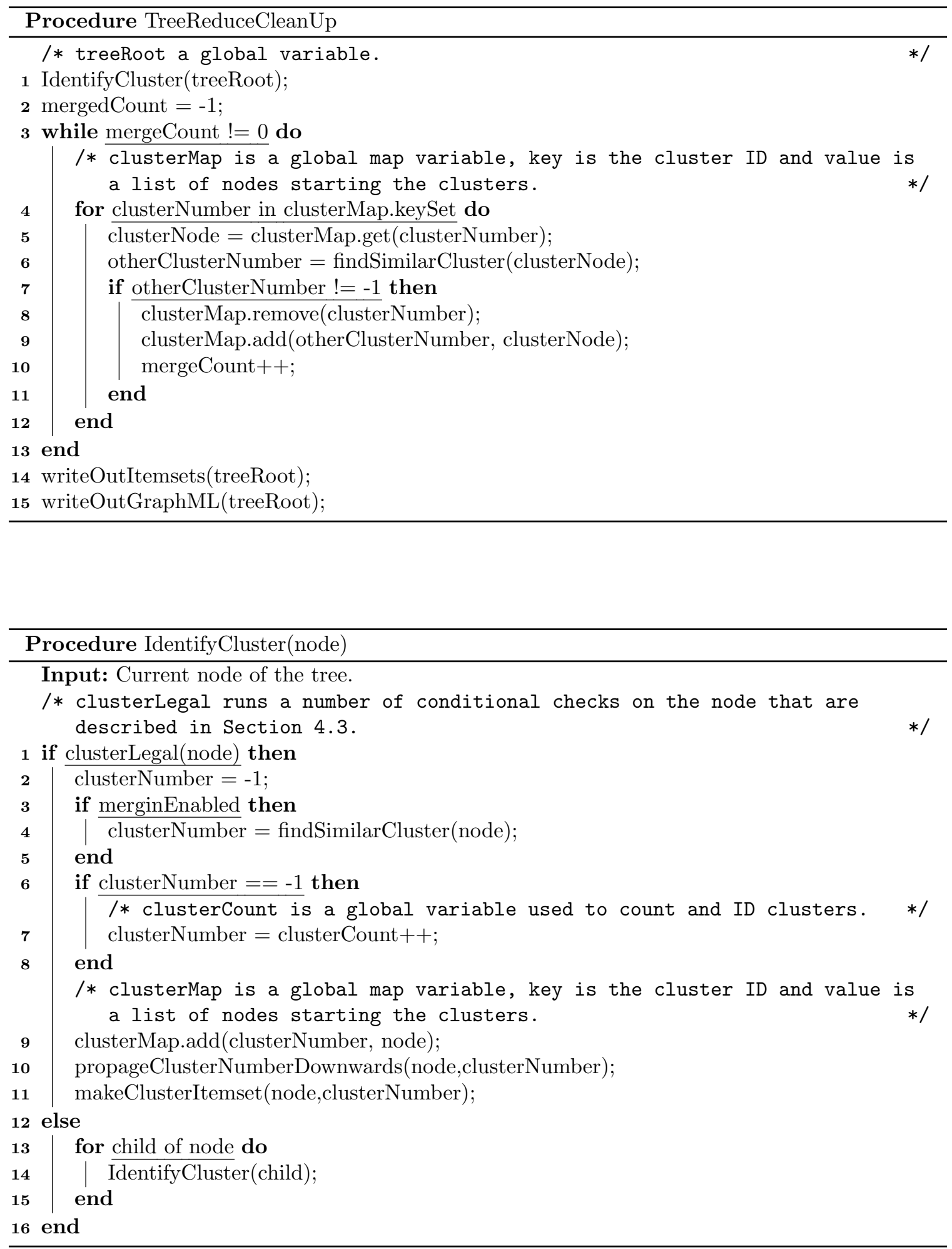


\section{A.6 Splinter Pseudocode}

The following is the pseudocode Splinter. The pseudocode for the script that parses the spam emails can be seen in Algorithm 1. Pseudocode of the script that detects campaigns is shown in Algorithm 2 and the accompanying Procedure IdentifyCampaigns.
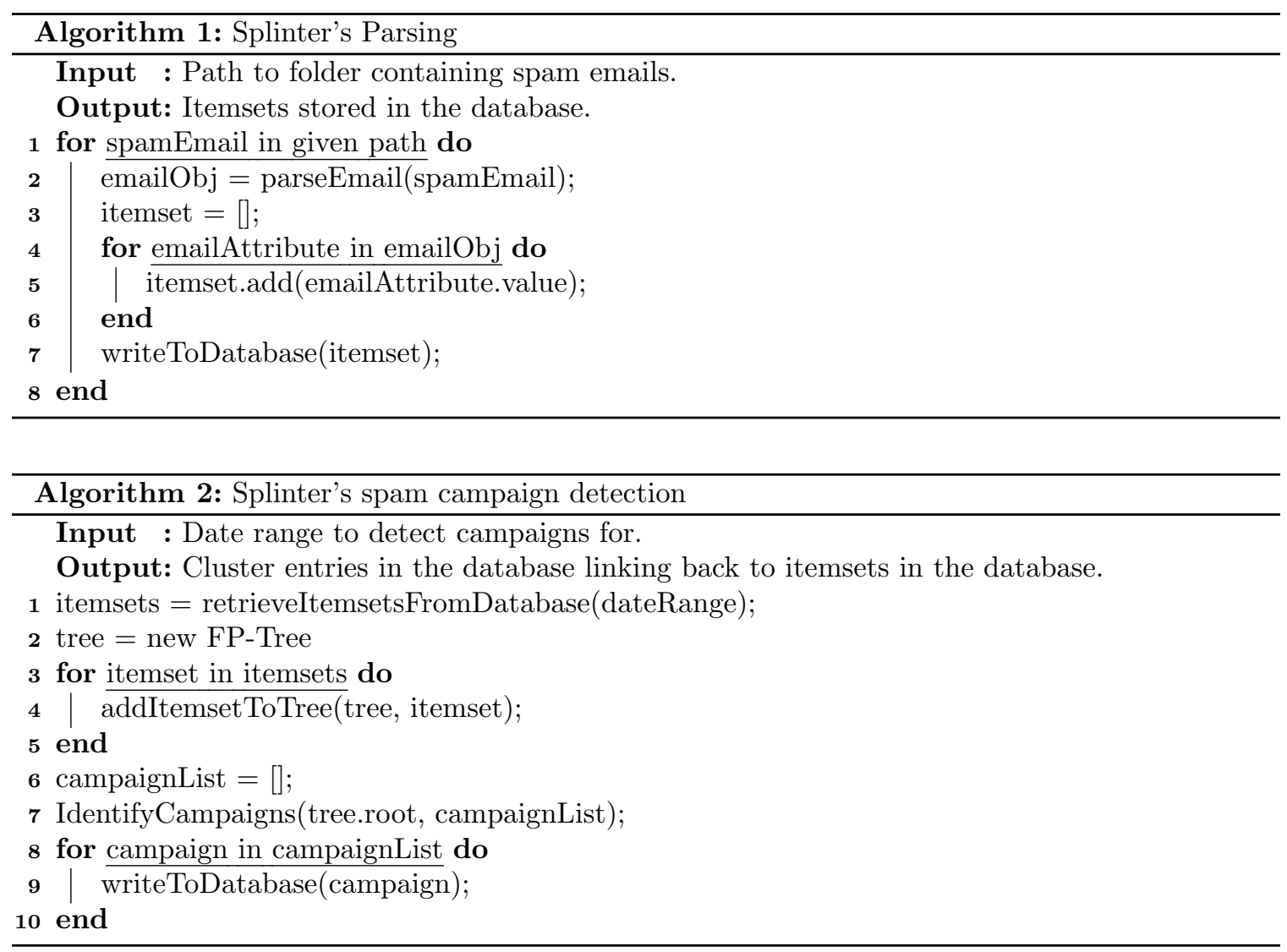


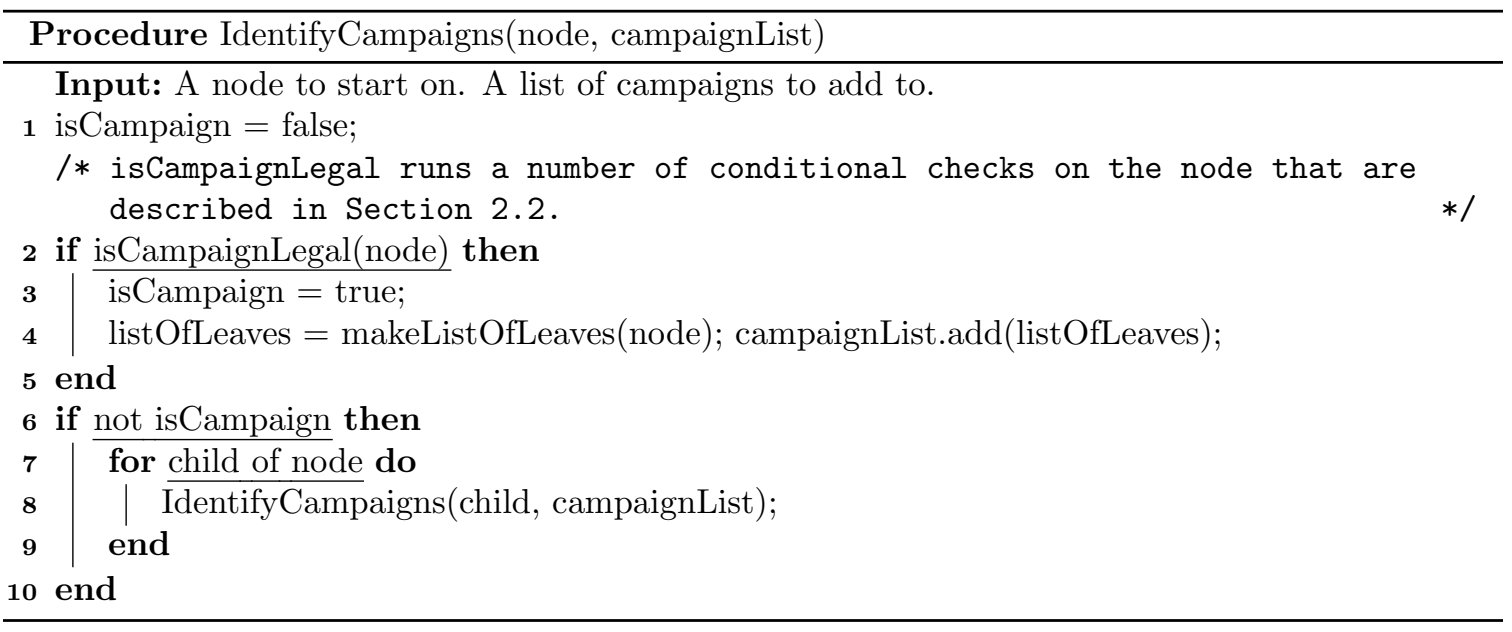




\section{Bibliography}

[1] Han, J., Pei, J., Yin, Y.: Mining frequent patterns without candidate generation. In: Proceedings of the 2000 ACM SIGMOD International Conference on Management of Data, New York, NY, USA, ACM (2000) 1-12

[2] Dinh, S., Azeb, T., Fortin, F., Mouheb, D., Debbabi, M.: Spam Campaign Detection, Analysis, and Investigation. International Journal of Digital Investigation 12 (2015) S12-S21

[3] Gudkova, D.: Spam in Q3 2013. https://securelist.com/analysis/ quarterly-spam-reports/57887/spam-in-q3-2013 (2013) Accessed: 2016-01-31.

[4] Tatyana Shcherbakova, Maria Vergelis, N.D.: Spam and phishing in the Q3 of 2014. https://securelist.com/analysis/quarterly-spam-reports/67851/ spam-and-phishing-in-the-q3-of-2014 (2014) Accessed: 2016-01-31.

[5] Tatyana Shcherbakova, Maria Vergelis, N.D.: Spam and phishing in Q3 2015. https://securelist.com/analysis/quarterly-spam-reports/72724/ spam-and-phishing-in-q3-2015 (2015) Accessed: 2016-01-31.

[6] Calais, P., Pires, D.E., Neto, D.O.G., Meira Jr, W., Hoepers, C., Steding-Jessen, K.: A campaign-based characterization of spamming strategies. In: CEAS 2008 - The Fifth Conference on Email and Anti-Spam, 21-22 August 2008, Mountain View, California, USA. Volume 8. (2008) $1-6$ 
[7] Md Shoeb, A.A., Mukhopadhyay, D., Al Noor, S., Sprague, A., Warner, G.: Spam Campaign Cluster Detection Using Redirected URLs and Randomized Sub-Domains. (2015)

[8] Borthakur, D.: HDFS Architecture Guide. https://hadoop.apache.org/docs/r1.2.1/hdfs_ design.html (2013) Accessed: 2016-04-18.

[9] Jaccard, P.: Étude comparative de la distribution florale dans une portion des alpes et des jura. Bulletin del la Société Vaudoise des Sciences Naturelles 37 (1901) 547-579

[10] Wikipedia: Overlap coefficient. https://en.wikipedia.org/w/index.php?title=0verlap_ coefficient\&oldid=666714979 (2015) Accessed: 2016-01-31.

[11] Rousseeuw, P.J.: Silhouettes: A graphical aid to the interpretation and validation of cluster analysis. Journal of Computational and Applied Mathematics 20 (1987) 53 - 65

[12] Levenshtein, V.I.: Binary codes capable of correcting deletions, insertions and reversals. In: Soviet Physics Doklady. Volume 10. (1966) 707

[13] Needleman, S.B., Wunsch, C.D.: A general method applicable to the search for similarities in the amino acid sequence of two proteins. Journal of Molecular Biology 48 (1970) $443-453$ Accessed on: 2016-11-06.

[14] Winkler, W.E.: The state of record linkage and current research problems. Technical report, Statistical Research Division, U.S. Census Bureau (1999)

[15] Lloyd, S.: Least squares quantization in PCM. IEEE Transactions on Information Theory 28 (1982) 129-137 